\title{
Nonlinear elliptic systems and mean-field games
}

\author{
Martino Bardi and Ermal Feleqi
}

\begin{abstract}
We consider a class of quasilinear elliptic systems of PDEs consisting of $N$ Hamilton-Jacobi-Bellman equations coupled with $N$ divergence form equations, generalising to $N>1$ populations the PDEs for stationary Mean-Field Games first proposed by Lasry and Lions. We provide a wide range of sufficient conditions for the existence of solutions to these systems: either the Hamiltonians are required to behave at most linearly for large gradients, as it occurs when the controls of the agents are bounded, or they must grow faster than linearly and not oscillate too much in the space variables, in a suitable sense. We show the connection of these systems with the classical strongly coupled systems of HamiltonJacobi-Bellman equations of the theory of $N$-person stochastic differential games studied by Bensoussan and Frehse. We also prove the existence of Nash equilibria in feedback form for some $N$-person games.
\end{abstract}

Mathematics Subject Classification. 35Q91, 49N70, 91A10.

Keywords. Nonlinear elliptic systems, Stochastic differential games, N-person games, Nash equilibria, Mean-Field Games.

\section{Introduction}

This paper deals with systems of partial differential equations of the following type

$$
\left\{\begin{array}{l}
\mathcal{L}^{i} v_{i}+H^{i}\left(x, D v_{i}\right)+\lambda_{i}=V^{i}[m] \quad \text { in } Q:=\mathbb{T}^{d} \\
\mathcal{L}^{i^{*}} m_{i}-\operatorname{div}\left(g^{i}\left(x, D v_{i}\right) m_{i}\right)=0 \quad \text { in } Q \\
\int_{Q} m_{i}(x) d x=1, \quad m_{i}>0, \quad \int_{Q} v_{i}(x) d x=0, \quad i=1, \ldots, N
\end{array}\right.
$$

This work was partially supported by the Fondazione CaRiPaRo Project "Nonlinear Partial Differential Equations: models, analysis, and control-theoretic problems" and the European Project Marie Curie ITN "SADCO-Sensitivity Analysis for Deterministic Controller Design". The authors are members of the Gruppo Nazionale per l'Analisi Matematica, la Probabilità e le loro Applicazioni (GNAMPA) of the Istituto Nazionale di Alta Matematica (INdAM). 
where the unknowns are the constants $\lambda=\left(\lambda_{1}, \ldots, \lambda_{N}\right)$, the functions $v=$ $\left(v_{1}, \ldots, v_{N}\right)$ and the densities of probability measures $m=\left(m_{1}, \ldots, m_{N}\right)$, at least continuous on the $d$-dimensional torus $Q:=\mathbb{T}^{d}$. The operators

$$
\mathcal{L}^{i}:=-\operatorname{tr}\left(a^{i}(x) D^{2}\right), \quad i=1, \ldots, N
$$

are second-order uniformly elliptic with $\mathbb{Z}^{d}$-periodic and Lipschitz coefficients $a^{i}(\cdot)$, i.e., for some $\nu, C>0$,$$
a^{i}(x) \geq \nu I_{d}, \quad a^{i}(x)=a^{i}(x+k) \forall k \in \mathbb{Z}^{d}, \quad\left|a^{i}(x)-a^{i}(x+y)\right| \leq C|y| \forall x, y \in \mathbb{R}^{d},
$$

where $I_{d}$ is the identity $d \times d$ matrix, and

$$
\mathcal{L}^{i^{*}} v:=-\sum_{h, k} D_{h k}^{2}\left(a_{h k}^{i}(x) v\right)
$$

are their formal adjoints.

The Hamiltonians $H^{i}: \mathbb{R}^{d} \times \mathbb{R}^{d} \rightarrow \mathbb{R}$ are $\mathbb{Z}^{d}$-periodic in $x$ and satisfy either one of the following alternative sets of conditions.

C1. For all $i=1, \ldots, N, H^{i}=H^{i}(x, p)$ is locally Lipschitz, superlinear in $p$ uniformly in $x$, i.e.,

$$
\inf _{x \in Q}\left|H^{i}(x, p)\right| /|p| \rightarrow+\infty \quad \text { as }|p| \rightarrow \infty,
$$

and $\exists \theta^{i} \in(0,1), C>0$, such that

$$
\operatorname{tr}\left(a^{i}\right) D_{x} H^{i} \cdot p+\theta^{i}\left(H^{i}\right)^{2} \geq-C|p|^{2} \quad \text { for }|p| \text { large, and for a.e. } x \in Q .
$$

C2. For all $i=1, \ldots, N$, for some $\alpha \in(0,1), H^{i}$ is locally $\alpha$-Hölder continuous and grows at most linearly in $p$, i.e., for some $C_{1}, C_{2}>0$,

$$
\left|H^{i}(x, p)\right| \leq C_{1}|p|+C_{2} \quad \forall x \in Q, p \in \mathbb{R}^{d} .
$$

The operators $V^{i}:\left(W^{1, p}(Q) \cap P(Q)\right)^{N} \rightarrow C^{0, \alpha}(Q)$ with $p>d$ are continuous with respect to the uniform convergence and either uniformly bounded in $C^{0,1}$ norm, if condition $\mathrm{C} 1$ holds, or uniformly bounded in the sup norm if condition $\mathrm{C} 2$ holds (see Sect. 2 for the notations and a more precise statement of these assumptions).

Finally, we assume that

$$
\begin{aligned}
& g^{i}: Q \times \mathbb{R}^{d} \rightarrow \mathbb{R}^{d} \text { are measurable, locally bounded, } \\
& \quad \text { and continuous in } p .
\end{aligned}
$$

Under the above conditions we show the existence of a solution $\lambda_{i} \in$ $\mathbb{R}, v_{i} \in C^{2, \alpha}(Q), m_{i} \in W^{1, p}(Q)$, for all $1 \leq p<\infty, i=1, \ldots, N$, to the system (1), where $\alpha$ is the Hölder exponent appearing in condition $\mathrm{C} 2$ on $H^{i}$ if such condition holds, and it is any number in $(0,1)$ if condition $\mathrm{C} 1$ is assumed instead.

There are two main motivations for studying systems of the form (1). The first is the theory of stationary Mean-Field Games (briefly, MFG) as formulated by Lasry and Lions $[44,46]$. In fact, for $N=1, \mathcal{L}^{i}=-\Delta, H^{i}=H$ smooth and $g^{i}=D_{p} H$, (1) reduces to the stationary MFG PDEs introduced and studied in $[44,46]$. This is a model of the equilibrium distribution of a 
large population of identical agents whose dynamics is subject to white noise and seeking to minimise a given individual cost functional. The additive eigenvalue problem (1) arises when such functional is the long-time-average of some running cost, sometimes called ergodic cost. For $N>1$ the system (1) with $g^{i}=D_{p} H^{i}$ can be associated to Mean-Field Games with $N$ different populations of players, see Sect. 3.6 and $[2,25]$ for further motivations. Mean-Field Games were also introduced independently by Huang, Caines, and Malhamé and studied by different methods [41,42], see Sect. 3.6.

In case of Condition C1 our assumptions allow for several populations and a more general controlled dynamics for the generic agent of each population than in $[44,46]$, but they are in the same spirit. Namely, the drift velocity of each player can point in any direction of the state space and can be arbitrarily large with a large cost, similarly to problems of Calculus of Variations. This is reflected by the strong coercivity condition (3) on the Hamiltonians $H^{i}$. Condition $\mathrm{C} 2$, instead, is natural in Control Theory, where the set of controls is bounded and therefore so are drift velocities and costs; moreover no controllability of the underlying system is assumed because $H^{i}$ is not required to have any coercivity property. See Sect. 3 and in particular Sects. 3.4 and 3.5 for several examples. Therefore the main novelties of this paper are in the results under condition $\mathrm{C} 2$, whose proofs are also quite different from those of $[44,46]$.

The second motivation is the synthesis of Nash equilibria in feedback form for $N$-person stochastic differential games with cost functionals of ergodic type. This is usually based on the solution of a strongly coupled system of $N$ Hamilton-Jacobi-Bellman (briefly, HJB) equations, the system (55) in Sect. 3.1, following a classical observation of Friedman [32]. A systematic study of such nonlinear elliptic systems was pursued by Bensoussan and Frehse starting with [14], see [15] for problems with ergodic cost and their book [16] for more references. In $[44,46]$ Lasry and Lions propose instead a system of $N$ HJB and $N$ Kolmogorov-Fokker-Planck equations of the form (1) for games where the players are coupled only via the cost functionals. An advantage of this system is the weaker coupling among the HJB equations. They give an existence result in the case $\mathcal{L}^{i}=-\nu^{i} \Delta, \nu^{i}>0, H^{i}$ smooth, satisfying (3) and (4) with $C=0$, and $g^{i}=D_{p} H^{i}$, and show how to synthesize a feedback Nash equilibrium for the $N$-person game from the solution of such system. In Sect. 3.1 we show a more precise connection among the classical systems of $[14,15]$ and systems of the form (1), which is related to the adjoint methods for HamiltonJacobi equation explored by Evans [30]. In Sect. 3 we also generalise the result in $[44,46]$ on the synthesis of the equilibrium and give several examples of classes of differential games to which our abstract results apply. Also for these applications the main novelties of our results are in the case of Condition $\mathrm{C} 2$, since the elliptic system for $N$-person games with ergodic costs had been studied so far only for Hamiltonians quadratic in $p[15,16]$ (see, however, [4] for twoperson, zero-sum games, and [17] for $N$-person with different cost functionals).

We recall that the pioneering papers $[44,46]$ prove also the convergence, in a suitable sense, of $N$-person games to a Mean-Field Game with a single population as the number of players tends to infinity. Some estimates of the 
present paper were used by Feleqi [31] to prove the same result in the case of several interacting populations and under more general conditions.

The proof of the existence of solutions to (1) under condition C1 follows the suggestion in $[44,46]$ about getting a priori estimates for $D v_{i}$ by the Bernstein method (see also $[1,47,48]$ ). On the other hand, rather than relying on estimates for Bellman equations, we mostly use more classical local a priori estimates for linear equations. We also included some of their proofs for the reader's convenience when we were not able to find an explicit reference in the literature, in the attempt to make the paper reasonably self-contained and readable by a wide audience. The proof in this case allows some variants to conditions $\mathrm{C} 1$ and $\mathrm{C} 2$ and can be adapted, for instance, to Hamiltonian behaving like $\left|p_{1}\right|+\left|p_{2}\right|^{\gamma}, \gamma>1$, as $p=\left(p_{1}, p_{2}\right) \in \mathbb{R}^{d_{1}} \times \mathbb{R}^{d_{2}}$ tends to infinity, see Remark 2.4 and Example 3.11.

Under condition $\mathrm{C} 2$ the proof is different. We first study an elliptic system where the additive eigenvalue $\lambda_{i}$ in (1) is replaced by a zero-th order term $\rho^{i} v^{i}$ with $\rho^{i}>0$. The existence of solutions for such system is achieved under the more general natural growth condition

$$
\left|H^{i}(x, p)\right| \leq C_{1}|p|^{2}+C_{2} \quad \forall x \in Q, p \in \mathbb{R}^{d},
$$

using some deep estimates by Ladyzhenskaya and Uraltseva [43] and other tools of the elliptic theory [34]. This result is of independent interest because the system is associated to feedback Nash equilibria for some $N$-person stochastic differential games with discounted infinite-horizon cost functionals (see Sect. 3.3). Next we find solutions of (1) by letting $\rho^{i} \rightarrow 0$, which corresponds to the small-discount approximation of the ergodic control problem $[4,6]$. We remark that the results under condition $\mathrm{C} 2$, Theorems 2.5 and 2.6, hold without any regularizing property of the operators $V^{i}$, which can be local functions of $m(x)$, provided they are bounded.

Note also that we do not assume any monotonicity property of the operators $V^{i}$, so our results can be applied, for instance, to models of segregation [2] or aggregation [28]. On the other hand we do not address the uniqueness of the solution, which is known to hold if $N=1$ under a monotonicity condition on $V^{i}$ introduced by Lasry and Lions $[44,46]$. If $N>1$ one does not expect uniqueness, and explicit examples of non-uniqueness can be found in [2]. For some very special cases of uniqueness with two populations see Cirant $[25,26]$ (where Neumann boundary conditions are also treated).

We conclude with some bibliographical remarks. The existence of Nash equilibria for some stochastic $N$-person differential games was also proved by probabilistic methods, see, e.g., $[21,33]$ and the references therein. The difficulties arising from constraints on the controls in such games were treated by Mannucci [49] working on parabolic systems. For a general presentations of Mean-Field Games and their applications we refer to the lecture notes by Guéant, Lasry, and Lions [40] and Cardaliaguet [22], the survey paper [39] and the very recent books $[35,37]$ by Gomes and collaborators. For the case of local coupling terms $V[m]$ we refer to Gomes and his coworkers $[36,38,50]$ (single population), and to Cirant [26-29] (several populations and Neumann 
boundary conditions). Evolutive MFG were first studied in [45,46] and [41,42]. The justification of stationary MFG via long-time asymptotics is in [23] and numerical methods in [3], see also the references therein. For other recent contributions on MFG see also the courses of P.-L. Lions at Collège de France, the monograph [18], the two special issues of Dynamic Games and Applications $[10,11]$, and the very recent important paper [24] on the master equation and its application to the convergence of games with a large population to a MFG.

The paper is organized as follows. Section 2 contains the statements and proofs of the existence results for (1) and for related systems with zero-th order terms arising from discounted infinite horizon problems. In Sect. 3 we describe the connections of the system (1) with N-person and Mean-Field games. We also apply the results of Sect. 2 to show the existence of Nash equilibria for such games in the cases of long-time-average costs and discounted infinite horizon costs. Finally, the Appendix contains the proofs of some technical lemmas.

\section{Existence of solutions to elliptic systems}

We denote by $C(Q)$ the set of $\mathbb{Z}^{d}$-periodic continuous functions on $\mathbb{R}^{d}$, by $C^{k, \alpha}(Q), k \in \mathbb{N}, 0<\alpha \leq 1$, the set of $\mathbb{Z}^{d}$-periodic functions having $k$-th order derivatives which are $\alpha$-Hölder continuous, by $L^{p}(Q), 1 \leq p \leq \infty$, the set of $p$-summable Lebesgue measurable $\mathbb{Z}^{d}$-periodic on $Q$, by $W^{k, p}(Q), k \in \mathbb{N}$, $1 \leq p \leq \infty$, the Sobolev space of $\mathbb{Z}^{d}$-periodic functions having weak derivatives up to order $k$ which are $p$-summable on $Q$, and by $P(Q)$ the set of probability measures on $Q$.

We will use the notations $\operatorname{tr} b$ for the trace of a square matrix $b$, and $a \cdot b=\operatorname{tr} a b^{t},|b|:=(b \cdot b)^{1 / 2}$. The adjoint operator $\mathcal{L}^{i^{*}}$ will be interpreted in the sense of distributions:

$$
\left\langle\mathcal{L}^{i^{*}} v, \phi\right\rangle=\int_{Q} v \mathcal{L} \phi d x \quad \forall \phi \in C^{\infty}(Q) .
$$

In all existence results we assume $V^{i}$ are continuous in the following sense $\forall m_{n}, m \in\left(W^{1, p}(Q) \cap P(Q)\right)^{N}, \quad\left\|m_{n}-m\right\|_{\infty} \rightarrow 0 \Longrightarrow\left\|V^{i}\left[m_{n}\right]-V^{i}[m]\right\|_{\infty} \rightarrow 0$.

\subsection{The additive eigenvalue problem}

For the first result, under condition C1, we assume $V^{i}:\left(W^{1, p}(Q) \cap P(Q)\right)^{N} \rightarrow$ $C^{0,1}(Q), p>d$, are uniformly Lipschitz continuous, i.e.,

$$
\sup _{m \in\left(W^{1, p}(Q) \cap P(Q)\right)^{N}}\left\|V^{i}[m]\right\|_{C^{0,1}(Q)}<\infty .
$$

Theorem 2.1. Assume (2) and (6), $H^{i}$ satisfy condition $\mathrm{C} 1$, and $V^{i}$ verify (7) and (8). Then there exist $\lambda_{1}, \ldots, \lambda_{N} \in \mathbb{R}, v_{1}, \ldots, v_{N} \in C^{2, \alpha}(Q), m_{1}, \ldots, m_{N} \in$ $W^{1, p}(Q)$, for all $0<\alpha<1,1 \leq p<\infty$, which solve the system (1).

We need the following two lemmas for linear equations. We believe they are well-known, but for lack of a precise reference we give their proofs in the Appendix. 
Lemma 2.2. Let

$$
\mathcal{L}=-a_{h k}(x) D_{h k}+b_{h}(x) D_{h}
$$

be a second-order uniformly elliptic linear differential operator in $Q=\mathbb{T}^{d}$ with coefficients $a_{h k}, b_{h}, \in C^{\alpha}(Q), h, k=1, \ldots, d, 0<\alpha<1$. Then, for any $f \in C^{\alpha}(Q)$, the problem

$$
\left\{\begin{array}{l}
\mathcal{L} v+\lambda=f \\
\int_{Q} v(x) d x=0
\end{array}\right.
$$

has a unique solution $(v, \lambda) \in C^{2, \alpha}(Q) \times \mathbb{R}$. Moreover,

$$
\begin{aligned}
& |\lambda| \leq\|f\|_{\infty}, \\
& \|v\|_{C^{1, \alpha}(Q)} \leq C\|f\|_{\infty}, \\
& \|v\|_{C^{2, \alpha}(Q)} \leq C\|f\|_{C^{\alpha}(Q)}
\end{aligned}
$$

for some constant $C>0$ which depends only on (the coefficients of) $\mathcal{L}$.

Lemma 2.3. Let

$$
\mathcal{L}=-a_{h k}(x) D_{h k}, \quad g: Q \rightarrow \mathbb{R}^{d}
$$

be a (symmetric) second-order uniformly elliptic linear differential operator in $Q=\mathbb{T}^{d}$ with coefficients $a_{h k} \in C^{0,1}(Q), h, k=1, \ldots, d$, and a bounded measurable vector field, respectively. Then the problem

$$
\left\{\begin{array}{l}
\mathcal{L}^{*} m-\operatorname{div}(g(x) m)=0 \\
\int_{Q} m(x) d x=1
\end{array}\right.
$$

has a unique solution $m \in W^{1, p}(Q)$ for all $1 \leq p<\infty$. Moreover, $m$ is positive and

$$
\|m\|_{W^{1, p}(Q)} \leq C\left(\|g\|_{\infty}\right),
$$

for some constant $C\left(\|g\|_{\infty}\right)$ which depends (continuously) on $g$ only through $\|g\|_{\infty}$ (and also on $p$ and the coefficients $a_{h k}$ ).

Proof of Theorem 2.1. The proof is based on Schauder's fixed point theorem (see for instance [52, Theorem 4.1.1, p. 25] or [34, Corollary 11.2, p. 280]) and on a priori estimates for the gradients of $v_{i}$ that are obtained by Bernstein's method, as suggested in $[44,46]$.

We first assume, instead of (3) and (4), that the Hamiltonians are bounded, that is,

$$
\exists M>0 \quad \text { such that }\left|H^{i}(x, p)\right| \leq M \quad \forall i, x, p .
$$

Let

$$
\mathcal{B}=\left\{u=\left(u_{1}, \ldots, u_{N}\right) \in\left(C^{1, \alpha}(Q)\right)^{N}: \int_{Q} u d x=0\right\},
$$

which is a Banach space as a closed subspace of $C^{1, \alpha}(Q)^{N}$. We define an operator

$$
T: \mathcal{B} \rightarrow \mathcal{B}
$$


according to the scheme

$$
u \mapsto m \mapsto(v, \lambda) \mapsto v,
$$

as follows. Given $u=\left(u_{1}, \ldots, u_{N}\right) \in \mathcal{B}$, we plug it in place of $v$ in the second $N$ scalar linear equations of the system (1) and solve those equations for the unknowns $m=\left(m_{1}, \ldots, m_{N}\right)$, requiring that these unknowns satisfy conditions in the third line of (1). That these $\left\{m_{i}\right\}_{i=1}^{N}$ exist, are uniquely defined and have the required properties, is a consequence of Lemma 2.3. Then, with these $\left\{m_{i}\right\}$, we solve the $N$ scalar linear equations obtained from the first equations in (1) after plugging $u_{i}$ into $H^{i}$ in place of $v_{i}$, i.e., by solving the $N$ uncoupled linear equations

$$
\mathcal{L}^{i} v_{i}+H^{i}\left(x, D u_{i}\right)+\lambda_{i}=V^{i}[m]
$$

for the unknowns $v=\left(v_{1}, \ldots, v_{N}\right), \lambda=\left(\lambda_{1}, \ldots, \lambda_{N}\right) \in \mathbb{R}^{N}$. Since we require in addition that the $v_{i}$ have zero mean, then $v=\left(v_{1}, \ldots, v_{N}\right) \in C^{2, \alpha}(Q)$ and $\lambda=\left(\lambda_{1}, \ldots, \lambda_{N}\right) \in \mathbb{R}^{N}$ are uniquely defined, as a consequence of Lemma 2.2. We set $T u=v$. By (10), (12), (14), and a standard embedding theorem, $T$ is continuous and compact. Moreover, the $C^{1, \alpha}$-estimate (11) and the boundedness of $H^{i}$ and $V^{i}$ gives

$$
\|v\|_{C^{1, \alpha}(Q)} \leq C
$$

for some $C>0$ independent of $v$; thus $T(\mathcal{B})$ is bounded. Therefore, by Schauder's fixed point theorem, $T$ has a fixed point (in the convex hull of the closure of $T(\mathcal{B})$ ).

Now we turn to consider Hamiltonians $H^{i}$ that satisfy the assumptions of the theorem. We introduce the truncated Hamiltonians $H_{R}^{i}$ defined as follows

$$
H_{R}^{i}(x, p)=\left\{\begin{array}{ll}
H^{i}(x, p), & \text { if }|p| \leq R, \\
H^{i}\left(x, R \frac{p}{|p|}\right), & \text { if }|p|>R,
\end{array} \quad x \in Q, p \in \mathbb{R}^{d},\right.
$$

where the parameter $R>0$ is to be fixed in the sequel sufficiently large. Let $R_{1}>0$ be such that (4) is verified for all $x \in Q,|p| \geq R_{1}$. Then

$$
\inf _{x \in Q}\left(\left(\operatorname{tr} a^{i}\right) D_{x} H_{R}^{i} \cdot p+\theta^{i}\left(H_{R}^{i}\right)^{2}\right) \geq-C|p|^{2} \quad \text { for all } R \geq|p| \geq R_{1},
$$

with the same $\theta^{i}(i=1, \ldots, N)$ and $C$ as in (4). Clearly the $H_{R}^{i}$ are bounded and Lipschitz continuous. So let $\lambda_{1}^{R}, \ldots, \lambda_{N}^{R} \in \mathbb{R}, v_{1}^{R}, \ldots, v_{N}^{R} \in C^{2, \alpha}(Q)$, $m_{1}^{R}, \ldots, m_{N}^{R} \in W^{1, p}(Q)(0<\alpha<1,1 \leq p<\infty)$ be a solution of (1) with $H_{R}^{i}$ in the place of $H^{i}$.

The crucial step of the proof is an a priori estimate for $\left\|D v_{i}^{R}\right\|_{\infty}$ uniform in $R$, obtained by Bernstein's method. We drop the indices $i$ and $R$ in the following estimates. Let $w=D v$ and $\psi=(1 / 2)|w|^{2}$. We have these identities

$$
\begin{aligned}
D \psi & =w D^{2} v \\
D^{2} \psi & =\sum_{h} w_{h} D^{2} w_{h}+\left(D^{2} v\right)^{2} \\
\mathcal{L} \psi & =-a \cdot D^{2} \psi=-\sum_{h} w_{h}\left(a \cdot D^{2} w_{h}\right)-a \cdot\left(D^{2} v\right)^{2} .
\end{aligned}
$$


Let us assume temporarily that $u$ is of class $C^{3}$ and $a_{h k}, H, F$ are of class $C^{1}$ (clearly the truncation of $H$ above could have been done smoothly). We apply to the first equations in (1) the operator $w \cdot D$ and use (19), (17) to obtain

$$
\mathcal{L} \psi+a \cdot\left(D^{2} v\right)^{2}-\delta a \cdot D^{2} v+D_{x} H \cdot w+D_{p} H \cdot D \psi=G \cdot w,
$$

where

$$
\delta a:=\left(\delta a_{h k}\right)_{h, k=1, \ldots, d} \quad \text { with } \delta a_{h k}:=\sum_{l} D_{l} a_{h k} w_{l}
$$

and $G$ is a function whose $L^{\infty}$-norm does not exceed a universal constant which does not depend on $v$ (recall the assumptions on operators $V^{i}$ ). We use the following inequalities which are simple consequences of Cauchy-Schwarz inequality: for any $a, b$ symmetric matrices with $a \geq 0$ and $c, e \in \mathcal{M}^{d \times m_{i}}$

$$
(a \cdot b)^{2} \leq\left(a \cdot b^{2}\right) \operatorname{tr} a \text { and }\left(\operatorname{tr} c e^{t} b\right)^{2} \leq|e|^{2} c c^{t} \cdot b^{2} .
$$

Assume that

$$
a=\frac{1}{2} \sigma \sigma^{t},
$$

where the matrix $\sigma(x)$ is Lipschitz; such a decomposition is always possible for $a(x)$ is Lipschitz and its smallest eigenvalue (which is positive) is bounded away from zero uniformly for $x \in Q$. By (23) and (21) we have

$$
\delta a=(\delta \sigma) \sigma^{t} .
$$

Using this identity, the second of inequalities (22) and (23), we obtain

$$
\begin{aligned}
\delta a \cdot D^{2} v & \leq|\delta \sigma|\left(\sigma \sigma^{t} \cdot\left(D^{2} v\right)^{2}\right)^{1 / 2}=\sqrt{2}|\delta \sigma|\left(a \cdot\left(D^{2} v\right)^{2}\right)^{1 / 2} \\
& \leq \varepsilon a \cdot\left(D^{2} v\right)^{2}+\frac{1}{2 \varepsilon}|\delta \sigma|^{2}
\end{aligned}
$$

for any $0<\varepsilon<1$. On the other hand, using the first of inequalities (22) and the first equations in (1), we have

$$
\begin{aligned}
\left(a \cdot\left(D^{2} v\right)^{2}\right) \operatorname{tr} a & \geq\left(a \cdot D^{2} v\right)^{2}=(\mathcal{L} v)^{2} \\
& \geq(\lambda+H-V[m])^{2} \\
& \geq \omega H^{2}-c_{\omega}
\end{aligned}
$$

for any $0<\omega<1$ and a constant $c_{\omega}$ independent of $R$, The last inequality is obtained by the boundedness of operators $V$ and constants $\lambda$. In fact, looking at the minima and maxima of $v$ in the first equations of (1) we obtain

$$
|\lambda| \leq \sup _{x \in Q}(|H(x, 0)|+|V[m](x)|) .
$$

Multiplying (20) by tra, and using (24), (25), we get $(\operatorname{tr} a)\left(\mathcal{L} \psi+D_{p} H D \psi+D_{x} H \cdot w\right)+(1-\varepsilon) \omega H^{2} \leq(\operatorname{tr} a)\left(G \cdot w+\frac{1}{2 \varepsilon}|\delta \sigma|^{2}\right)+c_{\omega}$. 
If we choose $\varepsilon$ and $\omega$ such that $(1-\varepsilon) \omega>\theta$, where $\theta$ is the constant appearing in (16), using (16) we get

$$
\begin{aligned}
& (\operatorname{tr} a)\left(\mathcal{L} \psi+D_{p} H D \psi\right)+((1-\varepsilon) \omega-\theta) H^{2} \\
& \quad \leq(\operatorname{tr} a)\left(G \cdot w+\frac{1}{2 \varepsilon}|\delta \sigma|^{2}\right)+C|w|^{2}+c_{\omega}
\end{aligned}
$$

for $R \geq|w|>R_{1}$. At a maximum point of $\psi_{i}^{R}$, (now we reintroduce $i$ and $R$ in order to avoid any possible confusion), say $x_{R}^{i}$, taking into account that $\left|\delta \sigma^{i}\right|$ is at most linear in $w_{i}^{R}$, we have

$$
\left(\left(1-\varepsilon^{i}\right) \omega^{i}-\theta^{i}\right)\left(H_{R}^{i}\right)^{2}\left(x_{R}^{i}, w_{i}^{R}\left(x_{R}^{i}\right)\right) \leq C\left(\left|w_{i}^{R}\left(x_{R}^{i}\right)\right|^{2}+1\right)
$$

for some $C>0$ (independent of $R$ ) and $R \geq\left|w_{i}^{R}\left(x_{R}^{i}\right)\right| \geq R_{1}$. But by (3), the left-hand side above is superlinear in $\left|w_{i}^{R}\left(x_{R}^{i}\right)\right|$ and thus $\left|w_{i}^{R}\left(x_{R}^{i}\right)\right|$ must be bounded by some constant independent of $R$.

Thus, we have shown that

$$
\left\|D u_{i}^{R}\right\|_{\infty} \leq R_{2}
$$

for some $R_{2}>0$ independent of $R>R_{1}$. So if take any $R>\max \left\{R_{1}, R_{2}\right\}$ in (15), we discover that $\lambda_{1}^{R}, \ldots, \lambda_{N}^{R} \in \mathbb{R}, v_{1}^{R}, \ldots, v_{N}^{R} \in C^{2, \alpha}(Q), m_{1}^{R}, \ldots, m_{N}^{R} \in$ $W^{1, p}(Q)(0<\alpha<1,1 \leq p<\infty)$ is also a solution of the original system of PDEs (1).

To complete the proof under our general assumptions we observe that Eq. (20) still holds a.e. in $\mathbb{R}^{d}$. In fact $u \in W^{3, p}, p>n$ (by classical elliptic regularity theory for linear equations), hence $u$ is three times differentiable in the usual sense a.e. and also the coefficients, being Lipschitz continuous, are differentiable a.e. A sort of chain rule usable for our purposes holds by a result in [5], and the maximum principle to be used is that of [20]. Alternatively, and more simply, one can proceed by regularizing the data $\left\{\left(a_{h k}^{i}\right)_{1 \leq h, k \leq d}: i=1, \ldots, N\right\}, V^{i}[m]$, for $m \in P(Q)^{N}$, and $H^{i}$, for $i=1, \ldots, N$, via smooth approximations to the identity $\left\{\rho_{\varepsilon}\right\}_{\varepsilon>0},\left\{\hat{\rho}_{\varepsilon}\right\}_{\varepsilon>0}$ given by $\rho_{\varepsilon}(x)=\varepsilon^{-d} \rho_{1}(x / \varepsilon)$ and $\hat{\rho}_{\varepsilon}(x, p)=\rho_{\varepsilon}(x) \rho_{\varepsilon}(p)$ for all $x \in \mathbb{R}^{d}, p \in \mathbb{R}^{d}, \varepsilon>0$, where $\rho_{1}$ is some mollification kernel in $\mathbb{R}^{d}$ (that is, a nonnegative function of class $C^{\infty}$ with support in the unit ball $B$ of $\mathbb{R}^{d}$ and $\left.\int_{B} \rho_{1}(z) d z=1\right)$. Noting that $\left\|\rho_{\varepsilon} \star a_{h k}^{i}\right\|_{C^{1}(Q)} \rightarrow\left\|a_{h k}^{i}\right\|_{C^{0,1}(Q)},\left\|\rho_{\varepsilon} \star V^{i}[m]\right\|_{C^{1}(Q)} \rightarrow\left\|V^{i}[m]\right\|_{C^{0,1}(Q)}$ and $\inf D_{x}\left(\hat{\rho}_{\varepsilon} \star H^{i}\right) \rightarrow \operatorname{ess} \inf D_{x} H^{i}$ as $\varepsilon \rightarrow 0$ for all $h, k, i, m \in P(Q)^{N}$, one deduces estimates of type (29) with $R_{2}$ independent of $\varepsilon$ for $\varepsilon$ small enough.

Remark 2.4. (i) In dimension $d=1$ condition (4) is not needed. Note that $\frac{d^{2} v_{i}}{d x^{2}}$ is bounded from below if $H^{i}$ is bounded from below; since it has zero mean in $(0,1)$, it is bounded in $L^{1}$ norm. Therefore $\frac{d v_{i}}{d x}$ is bounded.

(ii) Theorem 2.1 still holds if (3) is weakened to

$$
\exists \nu^{i}>0 \text { such that } \liminf _{|p| \rightarrow \infty} \frac{\inf _{x \in Q}\left|H^{i}(x, p)\right|}{|p|}=\nu^{i}>0,
$$


provided that we substitute (4) with the stronger condition: $\exists \theta^{i}, \eta^{i} \in$ $(0,1)$ such that

$\liminf _{|p| \rightarrow \infty} \frac{1}{|p|^{2}} \inf _{x \in Q}\left(\theta^{i}(\operatorname{tr} a) D_{x} H^{i} \cdot p+\left(1-\theta^{i}\right) \theta^{i} \eta^{i}\left(H^{i}\right)^{2}-|p|^{2} \frac{\left(\operatorname{tr} a^{i}\right)\left|D \sigma^{i}\right|^{2}}{2}\right) \geq 0$,

where $a^{i}=(1 / 2) \sigma \sigma^{t}, D \sigma^{i}=\left(D \sigma_{h k}^{i}\right)$ is a matrix (whose entries are vectors) and $\left|D \sigma^{i}\right|^{2}=\sum_{h, k}\left|D \sigma_{h k}^{i}\right|^{2}$. To see this take $\varepsilon^{i}=\theta^{i}$ in (24), multiply (26) by $\theta^{i}$ and choose $\omega^{i}$ in (25) so that $\omega^{i}>\eta^{i}$. Let $s>0$, $R_{s}>0$ be such that the quantity under the liminf sign in the left-hand side of (31) is $>-s$ for all $|p| \geq R_{s}$. Noting that $\left|\delta \sigma^{i}\right| \leq\left|D \sigma^{i}\right|\left|w_{i}^{R}\right|$, we deduce

$$
\left(\omega^{i}-\eta^{i}\right)\left(1-\theta^{i}\right) \theta^{i}\left(H_{R}^{i}\right)^{2}\left(x_{R}^{i}, w_{i}^{R}\left(x_{R}^{i}\right)\right)-s\left|w_{i}^{R}\left(x_{R}^{i}\right)\right|^{2} \leq C\left(\left|w_{i}^{R}\left(x_{R}^{i}\right)\right|+1\right)
$$

for some $C>0$ (independent of $R$ and $s$ ) and for $R \geq\left|w_{i}^{R}\left(x_{R}^{i}\right)\right| \geq R_{s}$. Now if we choose $s>0$ so small that $s<\nu^{i}\left(\omega^{i}-\eta^{i}\right)\left(1-\theta^{i}\right) \theta^{i}$, by (30) we find that $\left|w_{i}^{R}\left(x_{R}^{i}\right)\right|$ is bounded uniformly in $R$.

(iii) The arguments of the proof of Theorem 2.1, with some obvious modifications, prove also the solvability of the problem

$$
\mathcal{L} v+\lambda+H(x, D v)=0
$$

for the unknowns $v \in C^{2}(Q), \lambda \in \mathbb{R}$, where $\mathcal{L}$ satisfies (2) and the Hamiltonian $H$ is locally Lipschitz continuous and satisfies (3) and (4) or, alternatively, any condition mentioned in the remarks above. This result is known for Dirichlet and Neumann boundary value problems with $H$ satisfying similar conditions, see [47]. If $\mathcal{L}$ is degenerate elliptic the existence of a viscosity solution $v \in C^{0,1}(Q)$ can be found in [48].

These existence results for (1), under condition $\mathrm{C} 1$, may be interpreted as follows. The Hamiltonians $H^{i}$ can grow arbitrarily provided that they "do not oscillate too much in $x$ ", which rigorously means that they should satisfy the technical condition (4); moreover, the operators $V^{i}$ must be regularizing, therefore necessarily non-local.

On the other hand, if instead the Hamiltonians have at most linear growth as in condition $\mathrm{C} 2$ of the Introduction, we do not need any additional assumption of the aforementioned type, but the uniform boundedness of $V^{i}$. More precisely, in the next result we assume $V^{i}:\left(W^{1, p}(Q) \cap P(Q)\right)^{N} \rightarrow C^{0, \alpha}(Q)$ ( $\alpha$ being the same as in Condition C2) and

$$
\sup _{m \in\left(W^{1, p}(Q) \cap P(Q)\right)^{N}}\|V[m]\|_{\infty}<\infty .
$$

Theorem 2.5. Assume (2) and (6), $H^{i}$ satisfy condition $\mathrm{C} 2$, and that $V^{i}$ verifies (7), (32). Then there exist $\lambda_{1}, \ldots, \lambda_{N} \in \mathbb{R}, v_{1}, \ldots, v_{N} \in C^{2, \alpha}(Q)$, $m_{1}, \ldots, m_{N} \in W^{1, p}(Q)$, for all $1 \leq p<\infty$, which solve the system (1).

The proof is obtained as a limit in a system of equations with zero-th order terms, based on Theorem 2.6, and it is therefore postponed to the next section. 


\subsection{Equations with zero-th order terms}

The next result holds under a condition much larger than $\mathrm{C} 2$, where we replace the linear growth in $p$ of the Hamiltonians with a quadratic growth, the socalled natural growth condition.

$\mathrm{C} 2^{*}$. For all $i=1, \ldots, N$, for some $\alpha \in(0,1), H^{i}$ is locally $\alpha$-Hölder continuous and for some $C_{1}, C_{2}>0$,

$$
\left|H^{i}(x, p)\right| \leq C_{1}|p|^{2}+C_{2} \quad \forall x \in Q, p \in \mathbb{R}^{d},
$$

Theorem 2.6. Assume (2) and (6), $H^{i}$ satisfy condition $\mathrm{C}_{2}{ }^{*}$, and that $V^{i}$ verifies (7), (32). Let $\rho^{1}, \ldots, \rho^{N}$ be positive constants. Then there exist $v_{1}, \ldots, v_{N} \in$ $C^{2, \alpha}(Q), m_{1}, \ldots, m_{N} \in W^{1, p}(Q)$, for all $1 \leq p<\infty, i=1, \ldots, N$, which solve

$$
\begin{cases}\mathcal{L}^{i} v_{i}+H^{i}\left(x, D v_{i}\right)+\rho^{i} v_{i}=V^{i}[m] & \text { in } Q, \\ \mathcal{L}^{i^{*}} m_{i}-\operatorname{div}\left(g^{i}\left(x, D v_{i}\right) m_{i}\right)=0 & \text { in } Q, \\ \int_{Q} m_{i}(x) d x=1, & m_{i}>0, i=1, \ldots, N,\end{cases}
$$
pendix.

For the proof we need the following lemma which is proved in the Ap-

Lemma 2.7. Let

$$
\mathcal{L}=-a_{h k}(x) D_{h k}+b_{h}(x) D_{h}+c(x)
$$

be a second-order uniformly elliptic linear differential operator in $Q=\mathbb{T}^{d}$ with coefficients $a_{h k}, b_{h}, c \in C^{\alpha}(Q), h, k=1, \ldots, d, 0<\alpha<1$. Assume also that $c>0$. Then, for any $f \in C^{\alpha}(Q)$, the equation

$$
\mathcal{L} v=f
$$

has one and only one solution $v \in C^{2, \alpha}(Q)$. Moreover, for some constant $C>0$ which depends only on (the coefficients of) $\mathcal{L}$,

$$
\|v\|_{C^{2, \alpha}(Q)} \leq C\|f\|_{C^{\alpha}(Q)} .
$$

Proof of Theorem 2.6. We define an operator

$$
\begin{aligned}
& T: C^{1, \alpha}(Q)^{N} \rightarrow C^{1, \alpha}(Q)^{N}, \\
& u \mapsto m \mapsto v,
\end{aligned}
$$

in the following way. Given $u=\left(u_{1}, \ldots, u_{N}\right)$, we solve the second $N$ equations in (34) with $u_{i}$ plugged into $g^{i}$ in place of $v_{i}$ and with the corresponding normalisation conditions, and find $m=\left(m_{1}, \ldots, m_{N}\right)$, see Lemma 2.3. With these $m_{i}$ and the $u_{i}$ plugged into the Hamiltonians $H^{i}, i=1, \ldots, N$, in place of $v_{i}$ we solve, by Lemma 2.7, the first $N$ linear equations of (34), that is,

$$
\mathcal{L}^{i} v_{i}+\rho^{i} v_{i}+H^{i}\left(x, D u_{i}\right)=V^{i}[m], \quad i=1, \ldots, N
$$

and find $v=\left(v_{1}, \ldots, v_{N}\right) \in C^{2}(Q)$; (actually we can say that $v_{i} \in C^{2, \alpha^{2}}(Q)^{N}$ but this is not important; notice that $v_{i}$ is solution to a linear equation $\mathcal{L}^{i} v_{i}=f^{i}$ with $f^{i}(x)=V^{i}[m](x)-H^{i}\left(x, D u_{i}\right)-\rho^{i} v_{i}(x)$ which is $\alpha^{2}$-Hölder continuous for $x \mapsto H^{i}\left(x, D u_{i}(x)\right)$ is a composition of two $\alpha$-Hölder continuous functions, while the coefficients of the uniformly elliptic operator $\mathcal{L}^{i}$ are Lipschitz continuous). 
We set $T u=v$.

It is standard to verify that $T: C^{1, \alpha}(Q) \rightarrow C^{1, \alpha}(Q), u \rightarrow T u$ is continuous and compact. By Schaefer's version of Leray-Schauder theorem (see [52, Theorem 4.3.2, p 29] or [34, Theorem 11.3, p. 280]), we need only look at the set of the fixed points of the operators $s T, 0 \leq s \leq 1$, that is,

$$
\left\{u \in C^{1, \alpha}(Q)^{N}: s T u=u \text { for some } 0 \leq s \leq 1\right\},
$$

and prove that it is bounded in $C^{1, \alpha}(Q)^{N}$.

To prove such an estimate first note that if $u=s T u$ for some $0 \leq s \leq 1$, then, by assumption (32),

$$
\left\|u_{i}\right\|_{\infty}=\frac{s}{\rho^{i}} \max _{x \in Q}\left(\left|H^{i}(x, 0)\right|+\left|V^{i}[m](x)\right|\right) \leq C
$$

for some $0<C<\infty$ independent of $u$ and $s$, as can be seen by looking at the extrema of $u_{i}$ which satisfies Eq. (37) with $u_{i}=v_{i}$ and $H^{i}, V^{i}[m]$ multiplied by $s$.

Next we combine the $L^{\infty}$ bound with a classical a priori interior estimate for the gradients of solutions of quasilinear elliptic equations [43, Theorem 3.1, p. 266].

To do this we rewrite the equations in divergence form, use the quadratic growth condition (33), and assume temporarily that the coefficients $a^{i}$ of the equations are of class $C^{1}$. Then we get

$$
\left\|u_{i}\right\|_{C^{1}(Q)} \leq C
$$

for some $C>0$ independent of $u$ and $s$. Indeed, one has only to check carefully that all the assumptions of [43, Theorem 3.1] are satisfied. The additional regularity of the coefficients can now be removed by approximation because the estimates depend only on the $L^{\infty}$-norm of the derivatives of the coefficients and not on their moduli of continuity.

Finally, we can apply [34, Theorem 8.32, p. 210] to the linear uniformly elliptic equations $\mathcal{L}^{i} u_{i}=f^{i}$ with $f^{i}(x)=V^{i}[m](x)-H^{i}\left(x, D u_{i}(x)\right)-\rho^{i} u_{i}(x)$, since the $f^{i}$ are bounded and the coefficients of $\mathcal{L}^{i}$ are Lipschitz, in order to deduce, taking into account (39),

$$
\left\|u_{i}\right\|_{C^{1, \alpha}(Q)} \leq C
$$

for some $C>0$ again independent of $u$ and $s$. Thus $T$ has at least one fixed point.

By classical Schauder regularity results for linear uniformly elliptic equations (see, e.g., Lemma 2.7) applied to the same equations $\mathcal{L}^{i} u_{i}=f^{i}$ as above, we deduce that actually $u \in C^{2, \alpha}(Q)$; we need only to notice that $x \mapsto f^{i}(x)=V^{i}[m](x)-H^{i}\left(x, D u_{i}(x)\right)-\rho u_{i}(x)$ are $\alpha$-Hölder continuous and the coefficients of $\mathcal{L}^{i}$ are Lipschitz continuous.

Remark 2.8. An alternative existence result for the system (34) can be stated under condition $\mathrm{C} 1$ instead of $\mathrm{C} 2 *$ if $(8)$ holds. This requires only a slight modification of the proof of Theorem 2.1. 
Now we prove Theorem 2.5 on the existence of solutions for the system of ergodic PDEs (1) under condition $\mathrm{C} 2$ by approximation with solutions of (34) with vanishing zero-th order coefficients $\rho^{i}$.

Proof of Theorem 2.5. Let $v_{1}^{\rho}, \ldots, v_{N}^{\rho} \in C^{2, \alpha}(Q), m_{1}^{\rho}, \ldots, m_{N}^{\rho} \in W^{1, p}(Q)$ $(1 \leq p<\infty, i=1, \ldots, N)$ be a solution of (34) with $\rho^{i}=\rho>0$. Such a solution exists by Theorem 2.6. By the comparison principle for the $i$-th equation we first get, for all $i$ and $\rho \in] 0,1]$,

$$
\left\|\rho v_{i}^{\rho}\right\|_{\infty} \leq C_{2}+\left\|V^{i}\right\|_{\infty}
$$

Let $\left\langle v_{i}^{\rho}\right\rangle=\int_{Q} v_{i}^{\rho} d x$ be the mean of $v_{i}^{\rho}$. The crucial estimate is

$$
\left\|v_{i}^{\rho}-\left\langle v_{i}^{\rho}\right\rangle\right\|_{\infty} \leq C
$$

for some $C>0$ independent of $\rho$, which we prove borrowing an idea from $[4,6]$. Assume by contradiction that there is a sequence $\rho_{n} \rightarrow 0$ such that the sequence $\varepsilon_{n}:=\left\|v_{i}^{\rho_{n}}-\left\langle v_{i}^{\rho_{n}}\right\rangle\right\|_{\infty}^{-1}$ converges to 0 . The function $\psi_{n}:=\varepsilon_{n}\left(v_{i}^{\rho_{n}}-\right.$ $\left.\left\langle v_{i}^{\rho_{n}}\right\rangle\right)$ satisfies

$$
\mathcal{L}^{i} \psi_{n}+\varepsilon_{n} H^{i}\left(x, \frac{D \psi_{n}}{\varepsilon_{n}}\right)+\rho_{n} \psi_{n}=\varepsilon_{n}\left(V^{i}[m]-\rho_{n}\left\langle v_{i}^{\rho_{n}}\right\rangle\right) .
$$

Then, by the linear growth condition (5) on $H^{i}$ and (40), there is a constant $K$ such that

$$
\begin{aligned}
& \mathcal{L}^{i} \psi_{n}-C_{1}\left|D \psi_{n}\right|+\rho_{n} \psi_{n} \leq K \varepsilon_{n} . \\
& \mathcal{L}^{i} \psi_{n}+C_{1}\left|D \psi_{n}\right|+\rho_{n} \psi_{n} \geq-K \varepsilon_{n} .
\end{aligned}
$$

Since $\mathcal{L}^{i}$ is uniformly elliptic and $\left\|\psi_{n}\right\|_{\infty}=1$ we can apply the estimates of Krylov-Safonov type as stated in Thm. 5.1 of [53]. By (42) $\psi_{n}$ satisfies a local maximum principle with constants depending only on $d, \nu,\left\|a^{i}\right\|_{\infty}, C_{1}, C_{2}$, and $\left\|V^{i}\right\|_{\infty}$, whereas by (43) $\psi_{n}$ satisfies a weak Harnack inequality with constants depending only on the same quantities. The combination of these two estimates with the classical Moser iteration technique (see, e.g., [53]) implies that the family $\left\{\psi_{n}\right\}$ is equi-Hölder continuous.

Extracting a subsequence, we get that $\psi_{n}$ converges uniformly to a function $\psi$. Note that $\|\psi\|_{\infty}=1$ and that, by choosing $x_{n} \in Q$ such that $\psi_{n}\left(x_{n}\right)=$ 0 and extracting a further subsequence, we get $\psi(\bar{x})=0$ for some $\bar{x} \in Q$. Moreover $\psi$ is a viscosity solution of

$$
\mathcal{L}^{i} \psi-C_{1}|D \psi| \leq 0
$$

Since $\mathcal{L}^{i}$ is uniformly elliptic and $\psi$ is periodic, we deduce from the strong maximum principle (see, e.g., [9]) that $\psi$ must be a constant, which is a contradiction.

We complete the proof by showing that there exists a sequence $\rho_{n} \rightarrow 0$ such that, for $w_{i}^{\rho}=v_{i}^{\rho}-\left\langle v_{i}^{\rho}\right\rangle$,

$$
\left(w_{i}^{\rho_{n}}, \rho_{n}<v_{i}^{\rho_{n}}>, m_{i}^{\rho_{n}}\right) \rightarrow\left(v_{i}, \lambda_{i}, m_{i}\right) \quad \text { in } C^{2}(Q) \times \mathbb{R} \times C(Q),
$$

where $\left(v_{i}, \lambda_{i}, m_{i}\right), i=1, \ldots, N$, is a solution of (1). 
Indeed, we note that $\left(w_{i}^{\rho}, m_{i}^{\rho}\right)$ solve the equations

$$
\begin{cases}\mathcal{L}^{i} w_{i}^{\rho}+H^{i}\left(x, D w_{i}^{\rho}\right)=V^{i}\left[\left(m_{1}^{\rho}, \ldots, m_{n}^{\rho}\right)\right]-\rho\left\langle v_{i}^{\rho}\right\rangle & \text { in } Q, \\ \mathcal{L}^{i *} m_{i}^{\rho}-d i v\left(g^{i}\left(x, D w_{i}^{\rho}\right) m_{i}^{\rho}\right)=0 & \text { in } Q:=\mathbb{T}^{d}, \\ \int_{Q} m_{i}^{\rho}(x) d x=1, & m_{i}^{\rho}>0, \forall i=1, \ldots, N .\end{cases}
$$

By known a priori estimates for quasilinear elliptic equations (more precisely, by [43, Theorem 3.1, Ch. 4, p. 266]), $\left\|D w_{i}^{\rho}\right\|_{\infty}$ can be bounded in terms of $\left\|w_{i}^{\rho}\right\|_{\infty}$ and the data, in particular of the supremum norm of $V^{i}\left[\left(m_{1}^{\rho}, \ldots, m_{n}^{\rho}\right)\right]-$ $\rho\left\langle v_{i}^{\rho}\right\rangle$, which is bounded uniformly in $\rho$ by the assumptions on $V^{i}$ and (40). Then, applying [34, Theorem 8.32, p. 210] we deduce

$$
\left\|w_{i}^{\rho}\right\|_{C^{1, \alpha}(Q)} \leq C
$$

for some $C \geq 0$ and $0<\alpha \leq 1$ independent of $\rho$. Next, by the classical Schauder estimates and assumptions (8)

$$
\left\|w_{i}^{\rho}\right\|_{C^{2, \alpha}(Q)} \leq C
$$

for some $C$ and $\alpha$ independent of $\rho$. On the other hand, by Lemma 2.3 and assumptions (2), (6), for all $1 \leq p<\infty$,

$$
\left\|m_{i}^{\rho}\right\|_{W^{1, p}(Q)} \leq C
$$

for $C \geq 0$ independent of small enough $\rho$. Since $C^{2, \alpha}(Q) \times W^{1, p}(Q)$ for $p$ large enough is compactly embedded into $C^{2}(Q) \times C(Q)$, the previous estimates (46), (47) and the fact that the set $\left\{\rho\left\langle v_{i}^{\rho}\right\rangle: \rho>0\right\}$ is bounded by (40), we can extract a sequence $\rho_{n} \rightarrow 0$ such that (44) holds true.

Remark 2.9. If we drop the requirement that $H^{i}$ and $V^{i}[m]$ be Hölder continuous from the hypotheses of Theorems 2.5, 2.6 and require instead that $H^{i}$ be just a Carathèodory function (measurable in the first variable, continuous in the second) and $V^{i}:\left(W^{1, p}(Q) \cap P(Q)\right)^{N} \rightarrow L^{\infty}(Q)$ satisfies (32), then we can still conclude the existence of a solution for system (34), but in this case we can only say that $v_{i} \in W^{2, p}(Q)$ for every $1 \leq p<\infty$ (and hence $v_{i} \in C^{1, \alpha}(Q)$ for every $0<\alpha<1$ ).

Example 2.10. Let $d<p<\infty$. An example of a coupling term $V^{i}$ that satisfies conditions (7), (8) (for $\alpha=1$ ) is given by

$$
V[m](x)=F(x, \eta * m(x)) * \eta(x),
$$

where $\eta$ is a smooth regularizing kernel (that is, $\eta$ is a smooth function, $\int_{Q}|\eta| d x<\infty$, and $\left.\sup _{x \in Q}|D \eta|<\infty\right)$ and $F: Q \times \mathbb{R}_{+}^{N} \rightarrow \mathbb{R}$ a continuous function.

Another example is given by

$$
V[m](x)=F\left(x, \int_{Q} k(x, z) d m(x)\right),
$$

where $k: Q \times Q \rightarrow \mathbb{R}$ is continuous and $\alpha_{1}$-Hölder continuous in the first variable uniformly in the second, while $F: Q \times \mathbb{R}^{N} \rightarrow \mathbb{R}$ is a $\alpha_{2}$-Hölder 
continuous function, for $0<\alpha_{1}, \alpha_{2} \leq 1$. Then conditions (7), (8) are satisfied with $\alpha=\alpha_{1} \alpha_{2}$.

Example 2.11. (Local and bounded $V$ ) Theorems 2.5 and 2.6 cover also terms $V^{i}$ without regularizing properties, such as local functions of the form

$$
V[m](x)=F(x, m(x))
$$

where $d<p<\infty$, and $F: Q \times \mathbb{R}_{+}^{N} \rightarrow \mathbb{R}$ is a bounded $\alpha_{0}$-Hölder continuous function for some $0<\alpha_{0} \leq 1$. Then assumption (7) is satisfied with $\alpha=$ $(1-d / p) \alpha_{0}$ and assumption (32) follows from the boundedness of $F$.

\section{Stochastic differential games}

As an application of the previous results, in this section we show the existence of Nash equilibria for a class of $N$-person stochastic differential games with infinite horizon, such that the state of each player evolves independently from the states of the other players and the only coupling comes through the costs. Games of this type arise in many engineering and economic problems $[41,42$, 46].

Consider a control system driven by the stochastic differential equations

$$
d X_{t}^{i}=f^{i}\left(X_{t}^{i}, \alpha_{t}^{i}\right) d t+\sigma^{i}\left(X_{t}^{i}\right) d W_{t}^{i}, \quad X_{0}^{i}=x^{i} \in \mathbb{R}^{d}, i=1, \ldots, N
$$

where $\left\{W_{t}^{i}\right\}_{i=1}^{N}$ are $N$ independent Brownian motions in $\mathbb{R}^{d}, d \geq 1, A^{i} \subseteq \mathbb{R}^{m}$ are closed,

$$
f^{i}: \mathbb{R}^{d} \times A^{i} \rightarrow \mathbb{R}^{d} \quad \sigma^{i}: \mathbb{R}^{d} \rightarrow \mathbb{R}^{d \times d}
$$

are continuous, $\mathbb{Z}^{d}$-periodic and Lipschitz continuous in $x$ uniformly in $\alpha$, the matrix $\sigma^{i}(x)$ is nonsingular for any value of $x, \alpha_{t}^{i}$ is an admissible control of the $i$-th player, that is, a stochastic process taking values in $A^{i}$ and adapted to $W_{t}^{i}$. In view of the assumed periodicity in $x^{i}$ of all data we will often consider functions as defined on $Q=\mathbb{T}^{d}$.

\subsection{N-person games with long-time-average cost}

Consider a game where the $i$-th player seeks to minimize the long-time-average or ergodic cost

$$
J^{i}\left(X, \alpha^{1}, \ldots, \alpha^{N}\right):=\liminf _{T \rightarrow+\infty} \frac{1}{T} E\left[\int_{0}^{T} L^{i}\left(X_{t}^{i}, \alpha_{t}^{i}\right)+F^{i}\left(X_{t}^{1}, \ldots, X_{t}^{N}\right) d t\right]
$$

where $X=\left(x^{1}, \ldots, x^{N}\right)$ is the initial position of the system (49). On the cost of the $i$-th player (50) we assume

$$
\begin{aligned}
& L^{i}: Q \times A^{i} \rightarrow \mathbb{R} \quad \text { continuous, } \\
& F^{i}: Q^{N} \rightarrow \mathbb{R} \quad \alpha \text {-Hölder continuous }
\end{aligned}
$$


for some $\alpha \in(0,1)$. Define

$$
\begin{aligned}
\mathcal{H}^{i}\left(x^{i}, p, \alpha\right) & :=-p \cdot f^{i}\left(x^{i}, \alpha\right)-L^{i}\left(x^{i}, \alpha\right), \\
H^{i}\left(x^{i}, p\right) & :=\sup _{\alpha \in A^{i}} \mathcal{H}^{i}\left(x^{i}, p, \alpha\right), \quad p \in \mathbb{R}^{d},
\end{aligned}
$$

and suppose the sup is attained in the definition of $H^{i}$ for all $x^{i}, p$. Moreover, set

$$
a^{i}:=\sigma^{i}\left(\sigma^{i}\right)^{t} / 2, \quad \mathcal{L}^{i}:=-a^{i} \cdot D^{2} .
$$

Following the classical theory initiated by Friedman [32] and continued by Bensoussan and Frehse [14-16], we look at the Nash equilibria for the maximization of the pre-Hamiltonians with parameters $x^{j}, p^{j} \in \mathbb{R}^{d}, j=1, \ldots, N$, namely,

$$
P^{i}\left(\alpha^{1}, \ldots, \alpha^{N}\right):=-\sum_{j=1}^{N} f^{j}\left(x^{j}, \alpha^{j}\right) \cdot p^{j}-L^{i}\left(x^{i}, \alpha^{i}\right) .
$$

Clearly $\left(\bar{\alpha}^{1}, \ldots, \bar{\alpha}^{N}\right)$ is a Nash equilibrium for the static game with payoffs $P^{1}, \ldots, P^{N}$ if and only if $\bar{\alpha}^{i} \in \operatorname{argmax}_{\alpha^{i}} \mathcal{H}^{i}\left(x^{i}, p^{i}, \alpha^{i}\right)$ and the value of $P^{i}$ at the equilibrium is

$$
P^{i}\left(\bar{\alpha}^{1}, \ldots, \bar{\alpha}^{N}\right)=H^{i}\left(x^{i}, p^{i}\right)-\sum_{j \neq i} f^{j}\left(x^{j}, \bar{\alpha}^{j}\right) \cdot p^{j} .
$$

Therefore the system of Bellman equations of ergodic type [15,16] associated to the game considered here is

$$
\begin{cases}\sum_{j=1}^{N} \mathcal{L}^{j} v_{i}+H^{i}\left(x^{i}, D_{x^{i}} v_{i}\right)+\lambda_{i}=F^{i}(X)+\sum_{j \neq i} f^{j}\left(x^{j}, \bar{\alpha}^{j}\right) \cdot D_{x^{j}} v_{i} & \text { in } \mathbb{R}^{d N}, \\ \bar{\alpha}^{i} \in \operatorname{argmax}_{\alpha^{i}} \mathcal{H}^{i}\left(x^{i}, D_{x^{i}} v_{i}, \alpha^{i}\right), & i=1, \ldots, N,\end{cases}
$$

where the unknowns are the constants $\lambda_{i}$ and the functions $v_{i}(X), i=1, \ldots, N$. Note that this system is strongly coupled via the terms $f^{j}\left(x^{j}, \bar{\alpha}^{j}\right)$ on the right hand side of the equation for $v_{i}$, because $\bar{\alpha}^{j}$ depends on $D_{x^{j}} v_{j}$.

Assume there exist functions $\bar{\alpha}^{i}: \mathbb{R}^{d} \times \mathbb{R}^{d} \rightarrow A^{i}$ such that

$$
\begin{aligned}
& \bar{\alpha}^{i}(x, p) \text { is a maximum point for } \alpha \rightarrow \mathcal{H}^{i}(x, p, \alpha) \quad \forall x, p, \\
& \bar{\alpha}^{i} \text { is locally Lipschitz and } \mathbb{Z}^{d} \text {-periodic in } x,
\end{aligned}
$$

and define

$$
g^{i}(x, p)=-f^{i}\left(x, \bar{\alpha}^{i}(x, p)\right), \quad i=1, \ldots N .
$$

Making this choice in (55) we get

$$
\sum_{j=1}^{N} \mathcal{L}^{j} v_{i}+H^{i}\left(x^{i}, D_{x^{i}} v_{i}\right)+\sum_{j \neq i} g^{j}\left(x^{j}, D_{x^{j}} v_{j}\right) \cdot D_{x^{j}} v_{i}+\lambda_{i}=F^{i}(X) \quad \text { in } \mathbb{R}^{d N},
$$

$i=1, \ldots, N$. By a classical verification argument [15], if $\left(v_{i}, \lambda_{i}\right) \in C^{2}\left(\mathbb{T}^{d N}\right) \times$ $\mathbb{R}, i=1, \ldots, N$, is a solution, then $\bar{\alpha}^{i}\left(\cdot, D_{x^{i}} v_{i}(\cdot)\right), i=1, \ldots, N$, is a Nash 
equilibrium feedback. More precisely, if one solves the stochastic differential equation

$$
d X_{t}^{i}=f^{i}\left(X_{t}^{i}, \bar{\alpha}^{i}\left(X_{t}^{i}, D_{x^{i}} v_{i}\left(X_{t}\right)\right)\right) d t+\sigma^{i}\left(X_{t}^{i}\right) d W_{t}^{i}, \quad X_{0}^{i}=x^{i} \in \mathbb{R}^{d}, i=1, \ldots, N .
$$

then $\bar{\alpha}_{t}^{i}:=\bar{\alpha}^{i}\left(X_{t}^{i}, D_{x^{i}} v_{i}\left(X_{t}\right)\right), i=1, \ldots, N$, is a Nash equilibrium for the cost functionals (50), and $\lambda_{i}, i=1, \ldots, N$, are the values of the game corresponding to such equilibrium.

We want to derive from (59) a different system of elliptic equations of the form (1) from which we can still synthesize a Nash equilibrium feedback.

We follow an idea introduced by Lasry and Lions in the seminal paper [44]. Given a solution of (59) consider the equilibrium process defined by (60). Let us assume that

$$
D_{x^{i}} v^{i} \text { depends only on } x^{i} \text { for all } i \text {. }
$$

Then the equations in (60) are decoupled and each of them defines a nondegenerate diffusion $X_{t}^{i}$ on the torus $\mathbb{T}^{d}$, which has a unique ergodic invariant measure with density $m_{i}$. It is known that each $m_{i} \in C^{2}\left(\mathbb{T}^{d}\right)$ solves the Kolmogorov-Fokker-Planck equation

$$
\mathcal{L}^{i^{*}} m_{i}-d i v_{x^{i}}\left(g^{i}\left(x^{i}, D_{x^{i}} v_{i}\right) m_{i}\right)=0, \quad \int_{Q} m_{i}(x) d x=1, \quad i=1, \ldots, N,
$$

where $\mathcal{L}^{i *}$ is the formal adjoint of $\mathcal{L}^{i}$.

The next result exploits the adjoint structure of (59) and (62) to prove that multiplying the $i$-th equation in (59) by $\prod_{j \neq i} m_{j}\left(x^{j}\right)$ and integrating over $Q^{N-1}$ with respect to $d x^{j}, j \neq i$, we arrive at

$$
\mathcal{L}^{i} v_{i}+H^{i}\left(x, D v_{i}\right)+\lambda_{i}=V^{i}[m] \text { in } \mathbb{R}^{d},
$$

where

$$
V^{i}[m](x)=\int_{Q^{N-1}} F^{i}\left(x^{1}, \ldots, x^{i-1}, x, x^{i+1}, \ldots, x^{N}\right) \prod_{j \neq i} m_{j}\left(x^{j}\right) d x^{j} .
$$

Note that (52) implies (7) for such $V^{i}$ and that it is easy to see that also (32) holds.

Proposition 3.1. Assume $\left(v_{i}, \lambda_{i}\right) \in C^{2}\left(\mathbb{T}^{d N}\right) \times \mathbb{R}, i=1, \ldots, N$, is a solution of (59) satisfying (61) and $m_{1}, \ldots, m_{N}$ solve (62). Then, for all $i$, $x \mapsto v_{i}\left(x^{1}, \ldots, x^{i-1}, x, x^{i+1}, \ldots, x^{N}\right)$ solves $(63)$ for all $x^{1}, \ldots, x^{N}$.

Proof. Multiply the $i$-th equation in (59) by $\prod_{j \neq i} m_{j}\left(x^{j}\right)$ and integrate over $Q^{N-1}$ with respect to $d x^{j}, j \neq i$. Observe that, for $k \neq i$, integrating by parts with respect to $x^{k}$ we get

$$
\begin{aligned}
& \int_{Q^{N-1}}\left(\mathcal{L}^{k} v_{i}+g^{k}\left(x^{k}, D_{x^{k}} v_{k}\right) \cdot D_{x^{k}} v_{i}\right) \prod_{j \neq i} m_{j}\left(x^{j}\right) d x^{j} \\
& \quad=\int_{Q^{N-2}} \int_{Q} v_{i}\left(\mathcal{L}^{k^{*}} m_{k}-d i v_{x^{k}}\left(g^{k}\left(x^{k}, D_{x^{k}} v_{k}\right) m_{k}\right)\right) d x^{k} \prod_{j \neq i, k} m_{j}\left(x^{j}\right) d x^{j} .
\end{aligned}
$$


Then (62) gives

$$
\int_{Q^{N-1}} \sum_{j \neq i}\left(\mathcal{L}^{j} v_{i}+g^{j}\left(x^{j}, D_{x^{j}} v_{j}\right) \cdot D_{x^{j}} v_{i}\right) \prod_{j \neq i} m_{j}\left(x^{j}\right) d x^{j}=0 .
$$

On the other hand, using the assumption (61) and $\int_{Q} m_{j}(x) d x=1$, we also have

$$
\int_{Q^{N-1}}\left(\mathcal{L}^{i} v_{i}+H^{i}\left(x^{i}, D v_{i}\right)+\lambda_{i}\right) \prod_{j \neq i} m_{j}\left(x^{j}\right) d x^{j}=\mathcal{L}^{i} v_{i}+H^{i}\left(x^{i}, D v_{i}\right)+\lambda_{i}
$$

Now considering again the $i$-th equation in (59) multiplied by $\prod_{j \neq i} m_{j}\left(x^{j}\right)$ and integrated over $Q^{N-1}$ with respect to $d x^{j}, j \neq i$, and plugging into it the last two identities, we get that $v_{i}$ restricted to the variable $x^{i}$ solves (63) with $V^{i}$ given by (64).

Remark 3.2. If the maximum point $\bar{\alpha}^{i}(x, p)$ of $\mathcal{H}^{i}(x, p, \cdot)$ is unique, then $H^{i}$ is differentiable with respect to $p$ and $D_{p} H^{i}(x, p)=-f^{i}\left(x, \bar{\alpha}^{i}(x, p)\right)=g^{i}(x, p)$. Then the resulting system of HJB-KFP equations can be written in the same form as in the Lasry-Lions papers [44,46], namely,

$$
\begin{cases}\mathcal{L}^{i} v_{i}+H^{i}\left(x, D v_{i}\right)+\lambda_{i}=V^{i}[m] & \text { in } Q, \\ \mathcal{L}^{i^{*}} m_{i}-\operatorname{div}\left(D_{p} H^{i}\left(x, D v_{i}\right) m_{i}\right)=0 & \text { in } Q, \\ \int_{Q} m_{i}(x) d x=1, \quad m_{i}>0, \quad \int_{Q} v_{i}(x) d x=0, & i=1, \ldots, N .\end{cases}
$$

These notations also show that the KFP equations are the linearizations of the HJB equations around their solution $v_{1}, \ldots, v_{N}$.

From the theory of the previous section we get the following existence result.

Corollary 3.3. In addition to (2) and the assumptions of this section suppose either that $H^{i}$ satisfies condition $\mathrm{C} 1$ or that $H^{i}$ verifies $\mathrm{C} 2$ with the same $\alpha$ as in (52). Then there exist $\lambda_{1}, \ldots, \lambda_{N} \in \mathbb{R}, v_{1}, \ldots, v_{N} \in C^{2, \alpha}(Q), m_{1}, \ldots, m_{N} \in$ $W^{1, p}(Q), 1 \leq p<\infty$, which solve (1) with $V^{i}$ and $g^{i}$ given by (64) and (58), respectively.

\subsection{Synthesis of Nash equilibria}

Next we prove a verification result that produces Nash feedback equilibria for the $N$-person differential game from any solution of the HJB-KFP system of PDEs. We recall that a feedback for the $i$-th player is a Lipschitz map $\alpha^{i}: \mathbb{R}^{d} \rightarrow$ $A^{i}$ that generates a process $X_{t}^{i}$ solving $d X_{t}^{i}=f^{i}\left(X_{t}^{i}, \alpha^{i}\left(X_{t}^{i}\right)\right) d t+\sigma^{i}\left(X_{t}^{i}\right) d W_{t}^{i}$, $X_{0}^{i}=x^{i}$, and an admissible control $\alpha_{t}^{i}=\alpha^{i}\left(X_{t}^{i}\right)$. As usual, when we say "we plug feedback controls $x \mapsto \alpha^{j}(x), j=1, \ldots, N$, in the functional $J^{i}$, $i=1, \ldots, N$ " we mean that we are plugging its associated admissible control $t \mapsto \alpha^{j}\left(X_{t}^{j}\right)$, where $t \mapsto\left(X_{t}^{j}\right)_{j=1}^{N}$ is the solution of the system of SDEs

$$
d X_{t}^{j}=f^{j}\left(X_{t}^{j}, \alpha^{j}\left(X_{t}^{j}\right)\right) d t+\sigma^{j}\left(X_{t}^{j}\right) d W_{t}^{j}, \quad X_{0}^{j}=x^{j} j=1, \ldots, N .
$$

A Nash equilibrium of the $N$-person game of Sect. 3.1 for the initial position $X=\left(x^{1}, \ldots, x^{N}\right)$ is a vector of admissible controls $\left(\bar{\alpha}^{1}, \ldots, \bar{\alpha}^{N}\right)$ such that 


$$
\begin{aligned}
& J^{i}\left(X, \bar{\alpha}^{1}, \ldots, \bar{\alpha}^{i-1}, \alpha^{i}, \bar{\alpha}^{i+1}, \ldots, \bar{\alpha}^{N}\right) \geq J^{i}\left(X, \bar{\alpha}^{1}, \ldots, \bar{\alpha}^{N}\right) \\
& \quad \text { for all } \alpha^{i} \text { admissible control. }
\end{aligned}
$$

Theorem 3.4. Let $\lambda_{i}, v_{i}, m_{i}, i=1, \ldots, N$ be a solution of the system (1) as in the preceding Corollary 3.3. Then

$$
\bar{\alpha}^{i}(x):=\bar{\alpha}^{i}\left(x, D v_{i}(x)\right), \quad x \in \mathbb{R}^{d}, i=1, \ldots, N,
$$

define a feedback which is a Nash equilibrium for all initial positions $X \in Q^{N}$ of the control system (49). In addition, for each $X=\left(x^{1}, \ldots, x^{N}\right)$,

$$
\begin{aligned}
\lambda_{i} & =J^{i}\left(X, \bar{\alpha}^{1}, \ldots, \bar{\alpha}^{N}\right) \\
& =\liminf _{T \rightarrow+\infty} \frac{1}{T} E\left[\int_{0}^{T} L^{i}\left(\bar{X}_{t}^{i}, \bar{\alpha}^{i}\left(\bar{X}_{t}^{i}\right)\right)+F^{i}\left(\bar{X}_{t}^{1}, \ldots, \bar{X}_{t}^{N}\right) d t\right],
\end{aligned}
$$

where $\bar{X}_{t}^{i}$ is the process associated to the feedback $\bar{\alpha}^{i}$ with $\bar{X}_{0}^{i}=x^{i}$.

Proof. We follow the outline of proof in $[44,46]$. Let us first check (67). The processes $\bar{X}_{t}^{i}$ are independent diffusions on the torus, so each of them has a unique ergodic invariant measure, which must be $m_{i}$, see Chapter 3 , Section 3 of [19] or [4]. Then the joint process $\left(\bar{X}_{t}^{1}, \ldots, \bar{X}_{t}^{N}\right)$ is ergodic with invariant measure $\prod_{i=1}^{N} m_{i}\left(x^{i}\right)$, and therefore the right hand side of (67) is

$J^{i}\left(X, \bar{\alpha}^{1}, \ldots, \bar{\alpha}^{N}\right)=\int_{Q} L^{i}\left(x, \bar{\alpha}^{i}(x)\right) d m_{i}(x)+\int_{Q^{N}} F^{i}\left(x^{1}, \ldots, x^{N}\right) \prod_{j=1}^{N} d m_{j}\left(x^{j}\right)$.

On the other hand, the Ito-Dynkin formula gives

$E\left[v_{i}\left(\bar{X}_{T}^{i}\right)-v_{i}\left(x^{i}\right)\right]=E\left[\int_{0}^{T}\left(-g^{i}\left(\bar{X}_{t}^{i}, D v_{i}\left(\bar{X}_{t}^{i}\right)\right) \cdot D v_{i}\left(\bar{X}_{t}^{i}\right)-\mathcal{L}^{i} v_{i}\left(\bar{X}_{t}^{i}\right)\right) d t\right]$

By (56) and (58)

$$
g^{i}(x, p) \cdot p=H^{i}(x, p)+L^{i}(x, \bar{\alpha}(x, p)),
$$

so the HJB equation (63) implies

$$
\begin{aligned}
& E\left[v_{i}\left(\bar{X}_{T}^{i}\right)-v_{i}\left(x^{i}\right)\right]=\lambda_{i} T-E\left[\int_{0}^{T} L^{i}\left(\bar{X}_{t}^{i}, \bar{\alpha}^{i}\left(\bar{X}_{t}^{i}\right)\right)\right. \\
& \left.\quad+\int_{Q^{N-1}} F^{i}\left(x^{1}, \ldots, x^{i-1}, \bar{X}_{t}^{i}, x^{i+1}, \ldots, x^{N}\right) \prod_{j \neq i} d m_{j}\left(x^{j}\right) d t\right] .
\end{aligned}
$$

We divide by $T$ and let $T \rightarrow \infty$. The left-hand side vanishes, whereas the right hand side tends to $\lambda_{i}-J^{i}\left(X, \bar{\alpha}^{1}, \ldots, \bar{\alpha}^{N}\right)$, which proves $(67)$.

Next we check that the feedback law (66) defines a Nash equilibrium. We change the control of the $i$-th player into an arbitrary admissible control $\alpha_{t}^{i}$ and get 


$$
\begin{aligned}
& \frac{1}{T} E {\left[v_{i}\left(X_{T}^{i}\right)-v_{i}\left(x^{i}\right)\right]=\frac{1}{T} E\left[\int_{0}^{T}\left(f^{i}\left(X_{t}^{i}, \alpha_{t}^{i}\right) \cdot D v_{i}\left(X_{t}^{i}\right)-\mathcal{L}^{i} v_{i}\left(X_{t}^{i}\right)\right) d t\right] } \\
& \geq \lambda_{i}-\frac{1}{T} E\left[\int_{0}^{T} L^{i}\left(X_{t}^{i}, \alpha_{t}^{i}\right)\right. \\
&\left.\quad+\int_{Q^{N-1}} F^{i}\left(x^{1}, \ldots, x^{i-1}, X_{t}^{i}, x^{i+1}, \ldots, x^{N}\right) \prod_{j \neq i} d m_{j}\left(x^{j}\right) d t\right] .
\end{aligned}
$$

By the ergodicity of the joint process $\left(\bar{X}_{t}^{1}, \ldots, \bar{X}_{t}^{i-1}, X_{t}^{i}, \bar{X}_{t}^{i+1}, \ldots, \bar{X}_{t}^{N}\right)$ the last term on the right-hand side tends to $J^{i}\left(X, \bar{\alpha}^{1}, \ldots, \bar{\alpha}^{i-1}, \alpha^{i}, \bar{\alpha}^{i+1}, \ldots, \bar{\alpha}^{N}\right)$ as $T \rightarrow \infty$, and then

$$
J^{i}\left(X, \bar{\alpha}^{1}, \ldots, \bar{\alpha}^{i-1}, \alpha^{i}, \bar{\alpha}^{i+1}, \ldots, \bar{\alpha}^{N}\right) \geq \lambda_{i}=J^{i}\left(X, \bar{\alpha}^{1}, \ldots, \bar{\alpha}^{N}\right)
$$

for all $X \in Q^{N}$.

\subsection{A class of $N$-person games with discounted costs}

Consider again the controlled stochastic dynamics (49) with the same assumptions on the regularity and periodicity of the drifts $f^{i}$ and diffusions $\sigma^{i}, i=$ $1, \ldots, N$. For any set of controls $\left(\alpha^{i}\right)_{i=1}^{N}$, the corresponding solution processes $X_{t}^{i}, i=1, \ldots, N$, are ergodic with invariant measures $m_{i}(x)=m_{i}\left(x ; \alpha_{i}\right)$, $x \in Q$, see, e.g., [19, Theorem 3.2., p. 373]. The same is true for the joint process $X_{t}=\left(X_{t}^{i}, \ldots, X_{t}^{N}\right)$ with invariant measure $\prod_{i=1}^{N} m_{i}\left(x^{i}\right)$.

Each player $i$ seeks to minimize the discounted cost functional

$$
J_{\rho^{i}}^{i}\left(X, \alpha^{1}, \ldots, \alpha^{N}\right)=E\left[\int_{0}^{\infty} e^{-\rho^{i} t}\left(L^{i}\left(X_{t}^{i}, \alpha_{t}^{i}\right)+V^{i}[m]\left(X_{t}^{i}\right)\right) d t\right] .
$$

Here $L^{i}, V^{i}, i=1, \ldots, N$, are the same as in (51), (64), respectively, and $\rho^{i}>0$ is the discount rate of the $i$-th player. Note that only the state of the $i$ th player appears explicitly in the cost, whereas the states of the other players influence it only through their invariant measure.

In order to find Nash points we are led to the system of PDEs (34) where the discount rates $\rho^{i}$ appear as coefficients of the zero-th order terms, and $\mathcal{L}^{i}$, $H^{i}$ are given by (54), (53), respectively. We assume (56), (57), and define as before $g^{i}$ by (58). Under the assumptions of either Theorem 2.6 or Remark 2.8 there exists a solution $v_{1}, \ldots, v_{N} \in C^{2}(Q), m_{1}, \ldots, m_{N} \in W^{1, p}(Q), 1 \leq p<\infty$ to (34). Then a verification theorem similar to Theorem 3.4 gives the existence of feedback Nash equilibria for this game.

Theorem 3.5. Let $\rho^{1}, \ldots, \rho^{N}>0$ be given discount rates. For any solution $v_{1}^{\rho^{1}}, \ldots, v_{N}^{\rho^{N}} \in C^{2}(Q), m_{1}, \ldots, m_{N} \in W^{1, p}(Q), 1 \leq p<\infty$, of the system (34), the feedback law $\bar{\alpha}^{i}(x):=\bar{\alpha}^{i}\left(x, D v_{i}^{\rho^{i}}(x)\right), i=1, \ldots, N$, provides a Nash equilibrium for all initial positions $X \in Q^{N}$. Moreover, for each $X=\left(x^{i}\right)_{i=1}^{N} \in$ $Q^{N}$, 


$$
\begin{aligned}
v_{i}^{\rho^{i}}\left(x^{i}\right) & =J_{\rho^{i}}^{i}\left(X, \bar{\alpha}^{1}, \ldots, \bar{\alpha}^{N}\right) \\
& =E\left[\int_{0}^{\infty} e^{-\rho^{i} t}\left(L^{i}\left(\bar{X}_{t}^{i}, \bar{\alpha}^{i}\left(\bar{X}_{t}^{i}\right)\right)+V^{i}[m]\left(\bar{X}_{t}^{i}\right)\right) d t\right],
\end{aligned}
$$

where $\bar{X}_{t}^{i}$ is the solution of (49) associated to the feedback $\bar{\alpha}^{i}$.

This theorem applies to a larger class of systems and cost functionals than Theorem 3.4, because here we are assuming only the quadratic growth condition $\mathrm{C}^{*}$ on the Hamiltonians instead of the linear growth $\mathrm{C} 2$.

\subsection{Examples with unconstrained controls}

In this section we consider $A^{i}=\mathbb{R}^{d}$ for all $i$ and the system affine in the control, i.e.,

$$
f^{i}(x, \alpha)=\varphi^{i}(x)+\sum_{k=1}^{d} \alpha_{k} f_{k}^{i}(x)=\varphi^{i}(x)+\Phi^{i}(x) \alpha,
$$

where $\Phi^{i}$ is a square matrix whose columns are the vector fields $f_{k}^{i}, k=$ $1, \ldots, d$, and all vector fields $\varphi^{i}, f_{k}^{i}$ are Lipschitz in $Q=\mathbb{T}^{d}$ (the $d$-dimensional torus). Then

$$
H^{i}(x, p):=-p \cdot \varphi^{i}(x)+\sup _{\alpha \in \mathbb{R}^{d}}\left\{-p \cdot \Phi^{i}(x) \alpha-L^{i}(x, \alpha)\right\} .
$$

Assume $L^{i}$ is Lipschitz in $x$, uniformly as $\alpha$ varies in any bounded subset, and

$$
\lim _{\alpha \rightarrow \infty} \inf _{x \in Q} L^{i}(x, \alpha) /|\alpha|=+\infty .
$$

Then the sup in the definition of $H^{i}$ is attained and

$$
H^{i}(x, p)=L^{i^{*}}\left(x,-\Phi^{i}(x)^{t} p\right)-p \cdot \varphi^{i}(x), \quad L^{i^{*}}(x, q):=\max _{\alpha \in \mathbb{R}^{d}}\left\{q \cdot \alpha-L^{i}(x, \alpha)\right\},
$$

i.e., $L^{i^{*}}(x, \cdot)$ is the convex conjugate of $L^{i}(x, \cdot)$. Moreover $H^{i}$ is locally Lipschitz in $x$ and $p$. Now the other conditions of the existence Theorems 2.1 or 2.6 for the elliptic systems, and of the verification Theorems 3.4 or 3.5 can be checked on the last expression of $H^{i}$, as we show in the following examples. Recall that (71) implies that $L^{i^{*}}$ is superlinear in $q$, i.e.,

$$
\lim _{q \rightarrow \infty} \inf _{x \in Q} L^{i^{*}}(x, q) /|q|=+\infty,
$$

so $H^{i}$ cannot satisfy the linear growth condition in $\mathrm{C} 2$ unless $\Phi^{i}(x) \equiv 0$. Next we give examples satisfying $\mathrm{C} 1$ or $\mathrm{C} 2 *$.

Example 3.6. If for a $\gamma>0$

$$
L^{i}(x, \alpha) \geq \gamma\left(|\alpha|^{2}-1\right),
$$

then $L^{i^{*}}(x, q) \leq|q|^{2} / \gamma+\gamma$ and

$$
\left|H^{i}(x, p)\right| \leq C\left(1+|p|^{2}\right), \quad \forall x \in Q, p \in \mathbb{R}^{d} .
$$

Therefore $H^{i}$ satisfy condition $\mathrm{C} 2 *$ and Theorem 2.6 can be used. If (56) and (57) hold, then all the assumptions of Theorem 3.5 are verified. 
In order to have the coercivity condition (3) of $\mathrm{C} 1$ we will assume the matrix $\Phi^{i}$ is uniformly nonsingular in the following sense:

$$
\exists \delta>0 \quad \text { such that } \quad\left|\Phi^{i}(x) p\right| \geq \delta|p|, \quad \forall p .
$$

Example 3.7. Assume (74) and $L^{i}$ Lipschitz in $x$ uniformly in $\alpha$. Then condition C1 holds. In fact $\left|H^{i}(x, p)\right| /|p| \rightarrow+\infty$ as $|p| \rightarrow \infty$, uniformly in $x$, by the formula (72) for $H^{i}$ combined with (73) and (74). Moreover it is known that, at any point where $L^{i^{*}}$ is differentiable (therefore for a.e. $x \in Q$ ), one can compute

$$
D_{x} L^{i^{*}}(x, q)=-D_{x} L^{i}\left(x, \tilde{\alpha}^{i}(x, q)\right),
$$

where $\tilde{\alpha}^{i}(x, q)$ is any value of $\alpha$ at which the max in the definition of $L^{i^{*}}(x, q)$ is attained, see, e.g., [8, Lemma II.2.11, p. 43]. Then $D_{x} L^{i^{*}}$ is bounded and therefore $D_{x} H^{i} \cdot p=O\left(|p|^{2}\right)$. On the other hand $\left(H^{i}\right)^{2} /|p|^{2} \rightarrow+\infty$ as $|p| \rightarrow \infty$, so the condition (4) in $\mathrm{C} 1$ holds for any choice of $\theta^{i}>0$. In conclusion, Theorem 2.1 and Remark 2.8 apply to this example.

In order to apply the verification Theorems 3.4 and 3.5 and find Nash equilibrium feedbacks for the stochastic differential games we must also check the conditions (56) and (57) on the existence of a Lipschitz argmax for the Hamiltonians. A sufficient condition for them is that $L^{i}$ be differentiable with respect to $\alpha$ and $D_{\alpha} L^{i}(x, \cdot)$ be invertible (a fact related to the strict convexity of $L^{i}$ in $\alpha$ ) and locally Lipschitz. Then the sup in the definition of $H^{i}$ is attained at a unique value

$$
\bar{\alpha}^{i}(x, p)=\left(D_{\alpha} L^{i}\right)^{-1}\left(x,-\Phi^{i}(x)^{t} p\right),
$$

which is a locally Lipschitz function of $x$ and $p$.

Next we give two examples where we can check all the conditions for the existence of a feedback Nash equilibrium and give a more explicit formula for it. In both we assume that $F^{i}$ are Lipschitz, so $V^{i}$ defined by (64) verify (8).

Example 3.8. Consider

$$
L^{i}(x, \alpha)=\alpha^{t} B^{i}(x) \alpha,
$$

with positive definite matrices $B^{i}(x)$ Lipschitz in $Q=\mathbb{T}^{d}$ (the $d$-dimensional torus), and the affine system (70). Then

$$
L^{i^{*}}(x, q)=q^{t} B^{i}(x)^{-1} q / 4,
$$

so $H^{i}$ grows at most quadratically in $p$ and satisfies condition $\mathrm{C} 2 *$. Moreover

$$
\bar{\alpha}^{i}(x, p)=-\frac{1}{2} B^{i}(x)^{-1} \Phi^{i}(x)^{t} p
$$

verifies (56) and (57), so Theorem 3.5 on games with discounted costs applies and the Nash equilibrium feedback is linear in $p=D v_{i}$.

Assume in addition that $\Phi^{i}$ is uniformly nonsingular $(74)$ and $B^{i}(\cdot)^{-1}$ is Lipschitz. Then condition $\mathrm{C} 1$ holds. In fact, for $\gamma>0$ such that $q^{t} B^{i}(x)^{-1} q \geq$ $\gamma|q|^{2}$ for all $x$ and $q$, we have

$$
H^{i}(x, p) \geq \gamma \delta^{2}|p|^{2}-\max \left|\varphi^{i}\right||p|
$$


so (3) is verified. Moreover $\left|D_{x} H^{i}\right| \leq C\left(|p|^{2}+1\right)$, thus $D_{x} H^{i} \cdot p=O\left(|p|^{3}\right)$ and also (4) is satisfied for any choice of $\theta^{i}>0$. Therefore Remark 3.2, Corollary 3.3, and Theorem 3.4 on games with ergodic costs apply and produce the same linear Nash equilibrium feedback as above.

Example 3.9. Consider

$$
L^{i}(x, \alpha)=c^{i}(x) \frac{|\alpha|^{\gamma}}{\gamma}
$$

for some Lipschitz $c^{i}>0$ and $\gamma>1$, and the affine system (70) with $\Phi^{i}$ uniformly nonsingular (74). Then

$$
L^{i^{*}}(x, q)=c^{i}(x)^{1 /(1-\gamma)}|q|^{\gamma /(\gamma-1)} \frac{\gamma-1}{\gamma},
$$

so we can compute $D_{x} H^{i}$ by (72) and see that (4) is satisfied for any $\theta^{i}>0$. Therefore Theorem 2.1 and Remark 2.8 apply to this case with $g^{i}$ given by (58). Moreover

$$
\bar{\alpha}^{i}(x, p)=c^{i}(x)^{1 /(1-\gamma)}|q|^{(2-\gamma) /(\gamma-1)} q, \quad q=-\Phi^{i}(x)^{t} p,
$$

satisfies (56) and (57), so also Remark 3.2 and Theorem 3.4 on games with ergodic costs apply, as well as Theorem 3.5 on games with discounted costs.

\subsection{Examples with constrained controls}

Assume first that the vector fields $f^{i}$ have the general form described at the beginning of Sect. 3 with all control sets $A^{i}$ bounded, and (51), (52) hold. Then the Hamiltonians defined by (53) satisfy the linear growth condition (5) because

$$
\left|H^{i}(x, p)\right| \leq \sup _{Q \times A^{i}}\left|f^{i}\right||p|+\sup _{Q \times A^{i}}\left|L^{i}\right|, \quad \forall x \in Q, p \in \mathbb{R}^{d} .
$$

If $L^{i}$ is $\alpha$-Hölder, for some $0<\alpha \leq 1$, in $x$, condition C2 is verified. Then, assuming (56) and (57), all the assumptions of Corollary 3.3 and Theorems 2.6, 3.4 , and 3.5 are verified.

The next is a simple example where the argmax of the Hamiltonians is a singleton and verifies (57).

Example 3.10. Consider the system

$$
f^{i}(x, \alpha)=\varphi^{i}(x)-\alpha, \quad A^{i}=\left\{\alpha \in \mathbb{R}^{d}:|\alpha| \leq R_{i}\right\}, \quad \forall i,
$$

with $\varphi^{i}$ Lipschitz, $R_{i}>0$, and the costs

$$
L^{i}(x, \alpha)=c^{i}(x) \frac{|\alpha|^{2}}{2},
$$

for some Lipschitz $c^{i}>0$. Then

$$
\begin{aligned}
H^{i}(x, p) & =\max _{|\alpha| \leq R_{i}}\left\{p \cdot \alpha-c^{i}(x) \frac{|\alpha|^{2}}{2}\right\}-p \cdot \varphi^{i}(x) \\
& = \begin{cases}|p|^{2} /\left(2 c^{i}(x)\right)-p \cdot \varphi^{i}(x), & \text { if }|p| \leq R_{i} c^{i}(x), \\
R_{i}|p|-c^{i}(x) R_{i}^{2} / 2-p \cdot \varphi^{i}(x), & \text { if }|p|>R_{i} c^{i}(x) .\end{cases}
\end{aligned}
$$


Moreover

$$
\bar{\alpha}^{i}(x, p)= \begin{cases}p / c^{i}(x), & \text { if }|p| \leq R_{i} c^{i}(x), \\ R_{i} p /|p|, & \text { if }|p|>R_{i} c^{i}(x)\end{cases}
$$

satisfies (56) and (57). Also Remark 3.2 applies and we have again an explicit formula for the Nash equilibrium feedback. Note that $H^{i}$ is unbounded from below if $\left\|\varphi^{i}\right\|_{\infty}>R_{i}$, in particular it does not satisfy the coercivity condition (30).

Note also that we can restrict further $A^{i}$ to controls such that some components are null, say $\alpha_{k}=0$ for $k=1, \ldots, d_{1}, d_{1} \leq d$. Then the Hamiltonian is linear with respect to $p_{k}, k=1, \ldots, d_{1}$, but it still satisfies the assumptions of Corollary 3.3 and Theorems 2.6, 3.4, and 3.5.

In the last example some controls are bounded and the others are unconstrained; the Hamiltonians satisfy neither condition $\mathrm{C} 1$ nor $\mathrm{C} 2$, but a solution of the system (1) exists by Remark 2.4(ii).

Example 3.11. Take $d_{1}, d_{2} \geq 0$ and such that $d_{1}+d_{2}=d$. Consider the system

$$
f^{i}(x, \alpha)=\varphi^{i}(x)-\alpha, \quad A^{i}=\left\{\left(\alpha_{1}, \alpha_{2}\right) \in \mathbb{R}^{d_{1}} \times \mathbb{R}^{d_{2}}:\left|\alpha_{1}\right| \leq R_{i}\right\}, \quad \forall i,
$$

with $\varphi^{i}$ Lipschitz, $R_{i}>0$, and the costs

$$
L^{i}(x, \alpha)=c^{i} \frac{\left|\alpha_{1}\right|^{2}}{2}+\frac{\left|\alpha_{2}\right|^{\gamma}}{\gamma},
$$

for $c^{i}>0$ and $\gamma>1$. We write $p=\left(p_{1}, p_{2}\right) \in \mathbb{R}^{d_{1}} \times \mathbb{R}^{d_{2}}$ and find that for $\left|p_{1}\right|>R_{i} c^{i}$ the Hamiltonian is

$$
H^{i}(x, p)=R_{i}\left|p_{1}\right|+(\gamma-1) \frac{\left|p_{2}\right|^{\gamma /(\gamma-1)}}{\gamma}-p \cdot \varphi^{i}(x)-c^{i} \frac{R_{i}^{2}}{2},
$$

so neither the superlinearity condition (3) nor the linear growth (5) are satisfied. Denote $\varphi^{i}=\left(\varphi_{1}^{i}, \varphi_{2}^{i}\right) \in \mathbb{R}^{d_{1}} \times \mathbb{R}^{d_{2}}$ and $x=\left(x_{1}, x_{2}\right) \in \mathbb{R}^{d_{1}} \times \mathbb{R}^{d_{2}}$. Then $H^{i}$ verifies the coercivity condition (30) if $\left\|\varphi_{1}^{i}\right\|_{\infty}<R_{i}$. Moreover, $H^{i}$ satisfies also (31) if $R_{i}$ is large enough with respect to $\left\|D_{x_{1}} \varphi_{1}^{i}\right\|_{\infty}$ and $\left\|D \sigma^{i}\right\|_{\infty}$, as it is easy to check (for instance, (31) holds for any $R_{i}>0$ if $\sigma^{i}$ is constant and $\left.D_{x_{1}} \varphi_{1}^{i}=0\right)$. By the calculations in the Examples 3.9 and 3.10 also (56) and (57) hold true. Therefore, by Remark 2.4(ii), the conclusions of Corollary 3.3 and Theorem 3.4 hold in this case as well.

\subsection{Stationary mean-field games}

For $N=1$ the system of PDEs (1) is associated to a stationary Mean-Field Game if the Hamiltonian has the form (53) related to the vector field $g$ by (58). For $N>1$ it is related to Mean-Field Games involving $N$ distinct homogeneous populations of agents. Each population has a large number of identical players, and each of the players has a controlled nonlinear dynamics affected by white noise and seeks to minimise his individual cost functional (the withe noises of different agents are independent). The function $m_{i}$ in system (1) describes the density of the distribution of the $i$-th population. 
For MFG models $V^{i}$ typically has the form $G^{i}\left(x, K_{1}^{i} * m_{1}, \ldots, K_{N}^{i} * m_{N}\right)$ with $K_{j}^{i}$ Lipschitz kernels on $Q$ and $G^{i}: Q \times \mathbb{R}^{N} \rightarrow \mathbb{R}$ locally Lipschitz, so that the general conditions of the Introduction are satisfied. If the Hamiltonians $H^{i}$ and the vector fields $g^{i}$ are, respectively, of the form (53) and (58) of Sect. 3.1, we have existence of a solution $\lambda_{i}, v_{i}, m_{i}, i=1, \ldots, N$, to (1) under the same assumptions of Corollary 3.3, but with $V^{i}$ verifying (7), (8) under condition C1 and only (7), (32) under condition C2.

Such solution can be used for the following verification theorem. Given a vector of probability densities $m$ consider a generic agent of the $i$-th population with dynamics (49) and cost functional

$$
\tilde{J}^{i}\left(X_{0}^{i}, \alpha^{i}, m\right):=\liminf _{T \rightarrow+\infty} \frac{1}{T} E\left[\int_{0}^{T} L^{i}\left(X_{t}^{i}, \alpha_{t}^{i}\right)+V^{i}[m]\left(X_{t}^{i}\right) d t\right],
$$

with $V^{i}$ as above.

Proposition 3.12. Let $\lambda_{i}, v_{i}, m_{i}, i=1, \ldots, N$ be a solution of the system (1) with $H^{i}, g^{i}, V^{i}$ as above. Then

$$
\bar{\alpha}^{i}(x):=\bar{\alpha}^{i}\left(x, D v_{i}(x)\right), \quad x \in \mathbb{R}^{d}, i=1, \ldots, N,
$$

is an optimal feedback for any agent of the $i$-th population for all initial positions $X_{0}^{i} \in Q$, i.e.,

$$
\tilde{J}^{i}\left(X_{0}^{i}, \bar{\alpha}^{i}, m\right) \leq \tilde{J}^{i}\left(X_{0}^{i}, \alpha^{i}, m\right) \text { for all admissible controls } \alpha^{i} .
$$

Moreover, the probability density of the position of the $i$-th agent using such feedback is $m_{i}$.

Proof. It is enough to use a standard verification theorem in ergodic control (whose proof is very similar to that of Theorem 3.4). In fact, the $i$-th HJB equation of the system (1) is the Bellman equation of such a control problem with cost functional $\tilde{J}^{i}$. The second statement follows from the $i$-th KFP equation of the system (1) and the choice of $g^{i}(58)$.

The meaning of the result is that a solution of (1) provides an equilibrium for the MFG in the sense that for the generic agent of the $i$-th population the optimal control $\bar{\alpha}^{i}$ produces a probability distribution of the agent that coincides with the distribution $m_{i}$ of the whole $i$-th population.

Let us recall the connections between Mean-Field Games and games with large populations of identical players.

- Suppose we have only one population made of $\tilde{N}$ identical players with dynamics $f^{i}(x, \alpha)=\alpha, \sigma^{i}(x)=\nu>0, L^{i}=L$ for all $i=1, \ldots, \tilde{N}$, coupled only via the running costs $F^{i}\left(x^{1}, \ldots, x^{\tilde{N}}\right)=V\left[\frac{1}{\tilde{N}-1} \sum_{j \neq i} \delta_{x_{j}}\right]\left(x^{i}\right)$ where $V$ is an operator on measures with the properties described in the Introduction and $\delta_{x_{j}}$ are Dirac measures, so the argument of $V$ is the empirical distribution of the players different from $i$. Let $\lambda_{i}^{\tilde{N}}, v_{i}^{\tilde{N}}, m_{i}^{\tilde{N}}, i=1, \ldots, \tilde{N}$ be the solution of (1) given by Corollary 3.3. Then Lasry and Lions [44] showed that this sequence of solutions has a convergent subsequence as 
$\tilde{N} \rightarrow \infty$ and that the limit of each convergent subsequence solves the MFG PDEs with $N=1$

$$
\left\{\begin{array}{l}
-\Delta v+H(x, D v)+\lambda=V[m] \quad \text { in } Q, \\
-\Delta m-\operatorname{div}\left(D_{p} H(x, D v) m\right)=0 \quad \text { in } Q, \\
\int_{Q} m(x) d x=1, \quad m>0, \quad \int_{Q} v(x) d x=0 .
\end{array}\right.
$$

For linear-quadratic models a stronger convergence was proved by completely different methods in $[7,12]$.

- The same kind of limit result for general dynamics and $N$ populations of identical players, as the number of players of each population goes to infinity, was proved by Feleqi [31] using the methods of [44] and the estimates of Sect. 2 of the present paper.

- A basic result of the approach to MFG by Huang, Caines and Malhame $[41,42]$ states that the optimal feedback synthesised from the MFG PDEs is an $\epsilon$-Nash equilibrium for the $\tilde{N}$-person game for $\tilde{N}$ large enough, see also $[22]$.

\section{Appendix: Proofs of some technical results}

Proof of Lemma 2.2. For any solution $(v, \lambda) \in C^{2, \alpha}(Q) \times \mathbb{R}$ of $(9)$, we immediately deduce (10) by looking at the extrema of $v$.

Uniqueness. Thus $f \equiv 0$ implies $\lambda=0$. On the other hand $v \equiv 0$ otherwise the strong maximum principle (e.g., [34, Theorem 3.5, p. 35]) would be contradicted.

Existence. Consider (9) with $\mathcal{L}_{0}=-\Delta$ instead of $\mathcal{L}$. It is clearly solvable for any $f \in C^{\infty}$ with $\lambda=\int_{Q} f d x$ and $v$ that can be determined by Fourier series. Moreover, by Bessel's identity one deduces $\left\|D^{\beta} v\right\|_{p} \leq\|f\|_{p}$ for all $2 \leq$ $p<\infty$ and all multiindex $\beta$ with length $|\beta| \leq 2$. By letting $p \rightarrow \infty$, this estimate holds also for $p=\infty$. Thus (9) with $\mathcal{L}_{0}$ instead of $\mathcal{L}$ is solvable for any $f \in C(Q)$ and the solution $v \in C^{2}(Q)$. Actually, by Schauder interior estimates (see [34, Theorem 4.8, p. 62]) $f \in C^{\alpha}(Q)$ implies $v \in C^{2, \alpha}(Q)$.

In order to apply a continuity method, see [34, Theorem 5.2, p. 75], and deduce the solvability of (9), we introduce the operators

$$
\begin{aligned}
& T_{i}: C^{2, \alpha}(Q) / \mathbb{R} \times \mathbb{R} \rightarrow C^{\alpha}(Q), \quad i=0,1, \\
& (u, \lambda) \rightarrow T_{i}(u, \lambda)=\mathcal{L}_{i} u+\lambda \quad \mathcal{L}_{1}, \equiv \mathcal{L} .
\end{aligned}
$$

Note that

$$
[v]_{2, \alpha, Q}=\sup _{x, y \in Q,|\beta|=2} \frac{\left|D^{\beta} v(x)-D^{\beta} v(y)\right|}{|x-y|^{\alpha}}
$$

is a norm in $C^{2, \alpha}(Q) / \mathbb{R}$ equivalent to the natural one. Define also $T_{s}=(1-$ $s) T_{0}+s T_{1}, 0 \leq s \leq 1$. These operators are clearly linear and bounded. $T_{0}$ is also an isomorphism of Banach spaces. We need only prove that they are bounded from below in order to finish. This requires a careful look at Schauder interior estimates, see [34, Theorem 6.2, p. 90]. Let $\Omega \supset Q$ be a ball of diameter $D$ containing $Q$ and having same center as $Q$. We need some notation from [34]. 
For any function $g$ differentiable as many times as needed, if $d_{x}:=\operatorname{dist}(x, \partial \Omega)$, $d_{x, y}:=\min \left\{d_{x}, d_{y}\right\}$, we set

$$
\begin{aligned}
{[g]_{2, \alpha, \Omega}^{*} } & =\sup _{x, y \in \Omega,|\beta|=2} d_{x, y}^{2+\alpha} \frac{\left|D^{\beta} g(x)-D^{\beta} g(y)\right|}{|x-y|^{\alpha}} \\
{[g]_{l, \alpha, \Omega}^{(j)} } & =\sup _{x, y \in \Omega,|\beta|=2} d_{x, y}^{j+l+\alpha} \frac{\left|D^{\beta} g(x)-D^{\beta} g(y)\right|}{|x-y|^{\alpha}} \\
{[g]_{l, \Omega}^{(j)} } & =\sup _{x \in \Omega,|\beta|=l} d_{x}^{l+j}\left|D^{\beta} g(x)\right|, \quad|g|_{i, \alpha, \Omega}^{(j)}=\sum_{l=1}^{i}[g]_{l, \Omega}^{(j)}+[g]_{i, \alpha, \Omega}^{(j)}
\end{aligned}
$$

for any nonnegative integers $i, j, l$. Let $T_{s}(v, \lambda)=f$. Then, by $[34$, Theorem 6.2 , p. 90],

$$
[v]_{2, \alpha, \Omega}^{*} \leq C\left(\|v\|_{C(\Omega)}+|f-\lambda|_{0, \alpha, \Omega}^{(2)}\right),
$$

where $C$ depends only on a constant of ellipticity of $\mathcal{L}_{s}=(1-s) \mathcal{L}_{0}+s \mathcal{L}_{1}$ which clearly can be taken to be independent of $s$, and at most linearly on

$$
\max _{h, k}\left\{\left|1-s+s a_{h k}\right|_{0, \alpha, \Omega}^{(0)},\left|s b_{h}\right|_{0, \alpha, \Omega}^{(1)}\right\} \leq D^{1+\alpha} \max _{h, k}\left\{\left\|a_{h k}\right\|_{\infty},\left\|b_{h}\right\|_{C^{\alpha}(Q)}\right\},
$$

where $D$ is the diameter of $\Omega$. Therefore, taking also into account (10), by (77) we obtain

$$
[v]_{2, \alpha, Q} \leq C \frac{D^{1+\alpha}}{(D-\sqrt{d})^{2+\alpha}}\left(\|v\|_{\infty}+D^{2+\alpha}\|f\|_{C^{\alpha}(Q)}\right)
$$

for some $C$ independent of $D$. Now it is sufficient to take an $\Omega$ with a suitably large diameter $D$ in order to obtain (12). Finally, the $C^{1, \alpha}$-estimate (11) follows from [34, Theorem 8.32, p. 210]. This concludes the proof of this lemma.

Remark 4.1. An alternative proof of the existence relies on the approximation by equations with zero-th order terms $\mathcal{L} v+\rho v=f$ with $\rho>0$. A solution $v^{\rho}$ exists by Lemma 2.7, and we can let $\rho \rightarrow 0$ following the proof of Theorem 2.5.

Proof of Lemma 2.3. That $m \in W^{1,2}(Q)$ exists, is unique and positive is proved, e.g., in [19, Theorem 3.4, 378] or [13, Theorem 4.2, p. 133, Theorem 4.3 , p. 136]. (In [13] only the case $\mathcal{L}=-\Delta$ is treated but the techniques adapt easily to our operator.) The fact that $m \in W^{1, p}(Q)$ is also known, but for lack of a reference we sketch a proof here based on ideas of [51]. Split the operator $\mathcal{L}=\mathcal{L}_{0}+R$ into a (formally) selfadjoint part $\mathcal{L}_{0}=-D_{k}\left(a_{h k}(x) D_{h}\right)$ and a reminder $R=D_{k} a_{a k} D_{h}$. We use the deep fact that

$$
\mathcal{L}_{0}: W^{2, p}(Q) / \mathbb{R} \rightarrow L^{p}(Q) / \mathbb{R}, \quad 1<p<\infty
$$

is an isomorphism of Banach spaces. Then, by duality and interpolation,

$$
\mathcal{L}_{0}: W^{1, p}(Q) / \mathbb{R} \rightarrow W^{-1, p}(Q) / \mathbb{R}, \quad 1<p<\infty
$$

is also an isomorphism of Banach spaces. So we need only show that $\mathcal{L}_{0} m \in$ $W^{-1, p}(Q)$ in order to conclude. 
Note that, by Sobolev's embedding lemma, $m \in L^{p}(Q)$, where $p>2$ is given by $1 / p=1 / 2-1 / d$ if $d \geq 3$, or, otherwise, for any $1 \leq p<\infty$. By the Eq. (13), we have $\mathcal{L}_{0} m=-R^{*} m+\operatorname{div}(\mathrm{gm})$. Hence, for any $\varphi \in C^{\infty}(Q)$,

$$
\begin{aligned}
\left|\left\langle\mathcal{L}_{0} m, \varphi\right\rangle\right| & =\left|\int_{Q} m D_{k} a_{h k} D_{h} \varphi d x-\int_{Q} m g \cdot D \varphi d x\right| \\
& \leq\left(\|g\|_{\infty}+C\right)\|m\|_{p}\|D \varphi\|_{p^{\prime}}
\end{aligned}
$$

for some $C>0$ (independent of $g), p^{\prime}=p /(p-1)$. Therefore, $\mathcal{L}_{0} m \in W^{-1, p}(Q)$ and $m \in W^{1, p}(Q)$. We are done if $d \leq 2$. Otherwise, again Sobolev's lemma implies that $m \in L^{p}(Q)$, where $p$ now is given by $1 / p=1 / 2-2 / d$ if $d \geq 5$, or, otherwise, for any $1 \leq p<\infty$. In the same manner we conclude that $m \in W^{1, p}(Q)$. Thus, by a bootstrap argument, we deduce that $m \in W^{1, p}(Q)$ for all $1 \leq p<\infty$.

Moreover, by the estimates above and the fact that $\mathcal{L}_{0}: W^{1, p}(Q) / \mathbb{R} \rightarrow$ $W^{-1, p}(Q) / \mathbb{R}$ is an isomorphism, we deduce that

$$
\|m\|_{W^{1, p}(Q)} \leq C_{1}\left(\|g\|_{\infty}+C_{2}\right)\|m\|_{p}
$$

for some $C_{1}, C_{2}>0$ independent of $g$. Taking also into account [13, Theorem 4.3 , p. 136] which states that $\delta_{1}<m<\delta_{2}$ for some constants $\delta_{1}, \delta_{2}>0$ that depend only on $\|g\|_{\infty}$ (and in our case also on the coefficients $a_{h k}$, in a way which we do not specify because we will not need it in the sequel) we obtain (14).

Proof of Lemma 2.7. Uniqueness is standard. For the existence, we use a continuity method, e.g., [34, Theorem 5.2, p. 75], and Schauder a priori estimates to reduce to the equation corresponding to a simpler operator, say

$$
\mathcal{L}_{0}=-\Delta+1 \text {. }
$$

That $\mathcal{L}_{0} v=f$ has a solution $v \in C^{\infty}(Q)$ for each $f \in C^{\infty}(Q)$ can be shown, e.g., by Fourier series. Moreover, $\mathcal{L}_{0} v=f$ implies $\|v\|_{\infty} \leq\|f\|_{\infty}$. Then, by a Schauder estimate, see [34, Theorem 4.8, p. 62], $\|v\|_{C^{2, \alpha}(Q)} \leq C\|f\|_{C^{\alpha}(Q)}$. Now for an arbitrary $f \in C^{\alpha}(Q)$ consider a sequence $\left\{f_{n}\right\} \subset C^{\infty}(Q)$ such that $f_{n} \rightarrow f$ in $C^{\alpha}(Q)$. The sequence of the corresponding solutions $\left\{v_{n}\right\}$ is Cauchy in $C^{2, \alpha}(Q)$ and its limit $v$ verifies $\mathcal{L}_{0} v=f$.

Next, introduce the family of operators

$$
\begin{aligned}
& \mathcal{L}_{s}: C^{2, \alpha}(Q) \rightarrow C^{\alpha}(Q), \quad 0 \leq s \leq 1, \\
& u \rightarrow \mathcal{L}_{s} u=(1-s) \mathcal{L}_{0} u+s \mathcal{L} u
\end{aligned}
$$

For all $u \in C^{2, \alpha}(Q)$, by looking at its extrema, we have

$$
\|u\|_{\infty} \leq \max \left\{1,\|1 / c\|_{\infty}\right\}\left\|\mathcal{L}_{s} u\right\|_{\infty}
$$

Combining this with the interior Schauder estimates, see [34, Theorem 6.2, p. 90], we obtain

$$
\|u\|_{C^{2, \alpha}(Q)} \leq C\left\|\mathcal{L}_{s} u\right\|_{C^{\alpha}(Q)}
$$

for all $u \in C^{2, \alpha}(Q), 0 \leq s \leq 1$ and some $C>0$ independent of $u$ and $s$. Since $\mathcal{L}_{0}$ is onto, by the method of continuity [34, Theorem 5.2, p. 75$], \mathcal{L}_{1}$ is also 
onto, which is what we wanted to prove. With these considerations we also proved (36).

\section{References}

[1] Achdou, Y.: Mean field games: additional notes, C.I.M.E. course (2011)

[2] Achdou, Y., Bardi, M., Cirant, M.: Mean Field Games models of segregation (2016). arXiv:1607.04453

[3] Achdou, Y., Camilli, F., Capuzzo-Dolcetta, I.: Mean field games: numerical methods for the planning problem. SIAM J. Control Optim. 50, 77-109 (2012)

[4] Alvarez, O., Bardi, M.: Ergodicity, stabilization, and singular perturbations for Bellman-Isaacs equations. Mem. Am. Math. Soc. 204, 1-88 (2010)

[5] Ambrosio, L., Dal Maso, G.: A general chain rule for distributional derivatives. Proc. Am. Math. Soc. 108(3), 691-702 (1990)

[6] Arisawa, M., Lions, P.-L.: On ergodic stochastic control. Commun. Partial Differ. Equ. 23(11-12), 2187-2217 (1998)

[7] Bardi, M.: Explicit solutions of some linear-quadratic mean field games. Netw. Heterog. Media 7, 243-261 (2012)

[8] Bardi, M., Capuzzo-Dolcetta, I.: Optimal Control and Viscosity Solutions of Hamilton-Jacobi-Bellman Equations. Birkhäuser, Boston (1997)

[9] Bardi, M., Da Lio, F.: On the strong maximum principle for fully nonlinear degenerate elliptic equations. Arch. Math. (Basel) 73, 276-285 (1999)

[10] Bardi, M., Caines, P.E., Dolcetta, I.C.: Preface: DGAA special issue on mean field games. Dyn. Games. App. 3(4), 443 (2013). doi:10.1007/s13235-013-0098-3

[11] Bardi, M., Caines, P.E., Dolcetta, I.C.: Preface: DGAA 2nd special issue on mean field games. Dyn. Games. App. 4(2), 107 (2014). doi:10.1007/s13235-014-0104-4

[12] Bardi, M., Priuli, F.S.: Linear-Quadratic $N$-person and mean-field games with ergodic cost. SIAM J. Control Optim. 52, 3022-3052 (2014)

[13] Bensoussan, A.: Perturbation Methods in Optimal Control. Wiley, Chichester (1988)

[14] Bensoussan, A., Frehse, J.: Nonlinear elliptic systems in stochastic game theory. J. Reine Angew. Math. 350, 23-67 (1984)

[15] Bensoussan, A., Frehse, J.: Ergodic Bellman systems for stochastic games in arbitrary dimension. Proc. R. Soc. Lond. Ser. A 449(1935), 65-77 (1995)

[16] Bensoussan, A., Frehse, J.: Regularity Results for Nonlinear Elliptic Systems and Applications. Springer, Berlin (2002)

[17] Bensoussan, A., Frehse, J.: On diagonal elliptic and parabolic systems with super-quadratic Hamiltonians. Commun. Pure Appl. Anal. 8(1), 83-94 (2009) 
[18] Bensoussan, A., Frehse, J.: Yam: Mean Field Games and Mean Field Type Control Theory. Springer, New York (2013)

[19] Bensoussan, A., Lions, J.-L., Papanicolaou, G.: Asymptotic Analysis for Periodic Structures. North-Holland Publishing Co., Amsterdam (1978)

[20] Bony, J.-M.: Principe du maximum dans les espaces de Sobolev. C. R. Acad. Sci. Paris Sér. A-B. 265, A333-A336 (1967)

[21] Borkar, V.S., Ghosh, M.K.: Stochastic differential games: occupation measure based approach. J. Optim. Theory Appl. 73(2), 359-385 (1992) [Errata corrige 88(1), 251-252 (1996)]

[22] Cardaliaguet, P.: Notes on Mean Field Games (from P-L. Lions' lectures at Collège de France) (2010)

[23] Cardaliaguet, P., Lasry, J.-M., Lions, P.-L., Porretta, A.: Long time average of mean field games with a nonlocal coupling. SIAM J. Control Optim. 51(5), 35583591 (2013)

[24] Cardaliaguet, P., Delarue, F., Lasry, J.-M., Lions, P.-L.: The master equation and the convergence problem in mean field games (2015). arXiv:1509.02505 (preprint)

[25] Cirant, M.: Nonlinear PDEs in ergodic control, mean field games and prescribed curvature problems. Ph.D. Thesis, University of Padova (2013)

[26] Cirant, M.: Multi-population mean field games systems with Neumann boundary conditions. J. Math. Pures Appl. 103(9), 1294-1315 (2015)

[27] Cirant, M.: A generalization of the Hopf-Cole transformation for stationary mean-field games systems. C. R. Math. Acad. Sci. Paris 353, 807-811 (2015)

[28] Cirant, M.: Stationary focusing Mean Field Games. Commun. Partial Differ. Equ. (to appear). http://arxiv.org/abs/1602.04231

[29] Cirant, M., Verzini, G.: Bifurcation and segregation in quadratic two-populations Mean Field Games systems. ESAIM Control Optim. Calc. Var. (to appear). http://arxiv.org/abs/1511.09343

[30] Evans, L.C.: Adjoint and compensated compactness methods for HamiltonJacobi PDE. Arch. Ration. Mech. Anal. 197, 1053-1088 (2010)

[31] Feleqi, E.: The derivation of ergodic mean field game equations for several populations of players. Dyn. Games Appl. 3, 523-536 (2013)

[32] Friedman, A.: Stochastic Differential Equations and Applications, vol. 2. Academic Press, New York (1976)

[33] Ghosh, M.K., Suresh, K.K.: A nonzero-sum stochastic differential game in the orthant. J. Math. Anal. Appl. 305(1), 158-174 (2005)

[34] Gilbarg, D., Trudinger, N.S.: Elliptic Partial Differential Equations of Second Order. Classics in Mathematics. Springer, Berlin (2001) (Reprint of the 1998 edition) 
[35] Gomes, D.A., Nurbekyan, L., Pimentel, E.A.: Economic models and meanfield games theory. Publicacões Matemáticas do IMPA. Instituto Nacional de Matemática Pura e Aplicada, Rio de Janeiro (2015)

[36] Gomes, D.A., Patrizi, S., Voskanyan, V.: On the existence of classical solutions for stationary extended mean field games. Nonlinear Anal. 99, 49-79 (2014)

[37] Gomes, D.A., Pimentel, E.A., Voskanyan, V.: Regularity theory for mean-field game systems. Springer Briefs in Mathematics. Springer (2016)

[38] Gomes, D.A., Sanchez Morgado, H.: A stochastic Evans-Aronsson problem. Trans. Am. Math. Soc. 366, 903-929 (2014)

[39] Gomes, D.A., Saúde, J.: Mean field games models - a brief survey. Dyn. Games Appl. 4(2), 110-154 (2014)

[40] Guéant, O., Lasry, J.-M., Lions, P.-L.: Mean field games and applications. In: Carmona, R.A., et al. (eds.) Paris-Princeton Lectures on Mathematical Finance 2010. Lecture Notes in Mathematics, vol. 2003, pp. 205-266. Springer, Berlin (2011)

[41] Huang, M., Caines, P.E., Malhamé, R.P.: Large population stochastic dynamic games: closed-loop McKean-Vlasov systems and the Nash certainty equivalence principle. Commun. Inf. Syst. 6, 221-251 (2006)

[42] Huang, M., Caines, P.E., Malhamé, R.P.: Large-population cost-coupled LQG problems with nonuniform agents: individual-mass behavior and decentralized $\epsilon$-Nash equilibria. IEEE Trans. Autom. Control 52, 1560-1571 (2007)

[43] Ladyzhenskaya, O.A., Ural tseva, N.N.: Linear and Quasilinear Elliptic Equations. Academic Press, New York (1968)

[44] Lasry, J.-M., Lions, P.-L.: Jeux à champ moyen. I. Le cas stationnaire. C. R. Acad. Sci. Paris 343, 619-625 (2006)

[45] Lasry, J.-M., Lions, P.-L.: Jeux à champ moyen. II. Horizon fini et contrôle optimal. C. R. Acad. Sci. Paris 343, 679-684 (2006)

[46] Lasry, J.-M., Lions, P.-L.: Mean field games. Jpn. J. Math. 2(1), 229-260 (2007)

[47] Lions, P.-L.: Résolution de problèmes elliptiques quasilinéaires. Arch. Ration. Mech. Anal. 74(4), 335-353 (1980)

[48] Lions, P.-L., Souganidis, P.E.: Homogenization of degenerate second-order PDE in periodic and almost periodic environments and applications. Ann. Inst. H. Poincaré Anal. Non Lináire 22(5), 667-677 (2005)

[49] Mannucci, P.: Nonzero-sum stochastic differential games with discontinuous feedback. SIAM J. Control Optim. 43(4), 1222-1233 (2004/2005)

[50] Pimentel, E.A., Voskanyan, V.: Regularity for second order stationary mean-field games. http://arxiv.org/abs/1503.06445

[51] Schechter, M.: On $L^{p}$ estimates and regularity. I. Am. J. Math. 85, 1-13 (1963) 
[52] Smart, D.R.: Fixed Point Theorems. Cambridge Tracts in Mathematics, vol. 66. Cambridge University Press, London (1974)

[53] Trudinger, N.: Comparison principles and pointwise estimates for viscosity solutions of nonlinear elliptic equations. Rev. Mat. Iberoam. 4, 453-468 (1988)

Martino Bardi and Ermal Feleqi

Dipartimento di Matematica

Università di Padova

via Trieste, 63

35121 Padua

Italy

e-mail: bardi@math.unipd.it

Ermal Feleqi

e-mail: feleqi@math.unipd.it

Present Address:

Ermal Feleqi

Mathematics Institute

Cardiff University

Senghennydd Road

Cardiff

CF24 4AG

UK

e-mail: FeleqiE@cardiff.ac.uk

Received: 2 January 2016.

Accepted: 9 July 2016. 